\title{
Study about Non-Linear Structures
}

\section{Stéphane Le Corre}

Ecole Polytechnique Fédérale de Lausanne, Lausanne, Switzerland

Email: stephane.lecorre@epfl.ch

How to cite this paper: Le Corre, S. (2020) Study about Non-Linear Structures. Open Access Library Journal, 7: e6726. https://doi.org/10.4236/oalib.1106726

Received: August 19, 2020

Accepted: October 27, 2020

Published: October 30, 2020

Copyright $\odot 2020$ by author(s) and Open Access Library Inc.

This work is licensed under the Creative Commons Attribution International License (CC BY 4.0).

http://creativecommons.org/licenses/by/4.0/

\begin{abstract}
This article proposes a new algebraic structure that goes beyond the vector space, named sector space. We show that this new space is associated with projective spaces as vector space is associated with affine spaces. Parallelly, we suggest two other algebraic structures which extend the notions of group and field. Notions of element not reducible to a point (extended object), of ternary structure (extension of the binary notion of the linear group) and of complementary association (bifocal construction and mixture) seem to be the keystone of this building. These structures are associated with invariance properties by translation, rotation and zoom.
\end{abstract}

\section{Subject Areas}

Mathematics: Algebra, Geometry

\section{Keywords}

Algebraic Structures, Geometrical Spaces, Projective Spaces, Non-Linear Structures (Space, Field and Group)

\section{Introduction}

In this article, we define a new geometrical space which extends the notion of vector to geometrical objects which we will name sector (portion of surface in the form of parallelogram). We will extract from it 3 propositions of algebraic structures which extend the notions of group, field and vector space. These structures will be associated with invariance properties by translation, rotation and zoom. They will make it possible to go beyond the binary limitations of linear structures, which is why we will qualify them as non-linear. It will result from it, notions of element not reducible to a point (extended object), of ternary structure (extension of the binary notion of the linear group) and of complementary association which will be the keystone of this building. We will 
see how this sectoral space is associated with projective spaces (in a similar way that vector space with affine spaces [1]).

\section{Sectoral Geometric Space}

\subsection{Basic Geometric Objects, Sectors}

We limit ourselves to a "minimal" 2-dimensional space (plane). We consider the set of parallelograms that we can define in this space. These plane portions are defined by 2 segments (blue and orange segment in the following figures) which orient the parallelogram. The orange side will be named the base/reference of the sector:

These objects will be named free sectors. Any orange side element of zero length will be excluded. These elements will therefore not be considered as sectors. The "flat" sectors for which the orange and blue side will be collinear will be elements of our space and the blue side can be zero. Figure 1 shows the examples of different sectors.

\subsection{Equivalence Classes on Sectors}

We are going to define object classes according to the following equivalence relation. 2 free sectors will be considered as 2 representatives of the same class if they can overlap exactly (with the same reference) by the combination of the operations of translation (not to depend on an absolute position), rotation (not to depend on an absolute direction), zoom, i.e. homothety of any center and of nonzero factor (not to depend on an absolute size).

In Figure 2, all these free sectors represent the same sector. (0) and (1) are deduced from each other by a translation. (0) and (2) are deduced from each other by a rotation (and a translation). (2) and (3) is deduced from each other by a homothety (zoom) (and a translation).

In Figure 3, the 2 free sectors (4) and (5) are different (they represent 2 different equivalence classes) because they cannot be superimposed by the 3
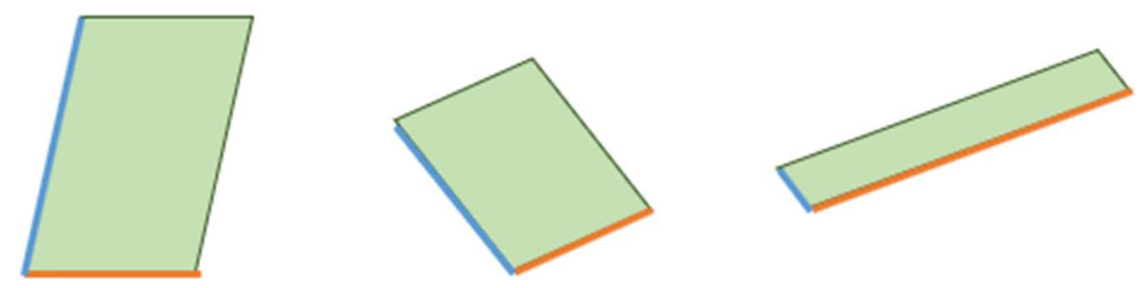

Figure 1. Examples of different sectors.

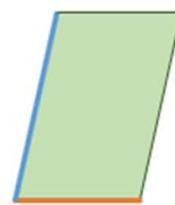

(0)

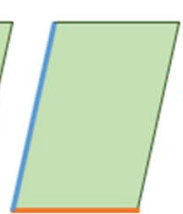

(1)

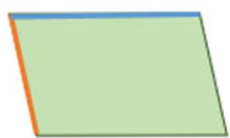

(2)

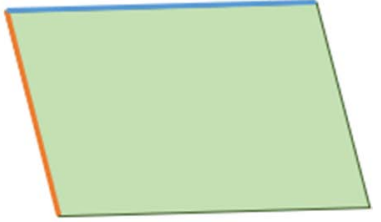

(3)

Figure 2. Four representations of a same sector. 


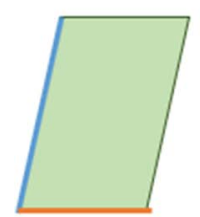

(4)

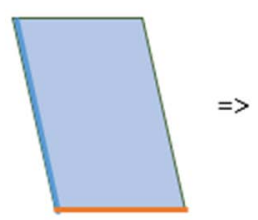

(5)

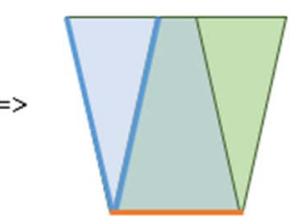

(6)

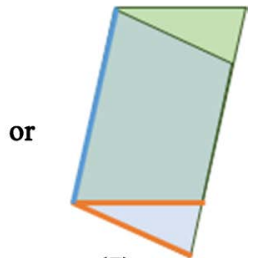

(7)

Figure 3. Two different classes of sector.

transformations of the equivalence class (translation, rotation, zoom). We see the result of these attempts when we try to either superimpose the orange sides (6) or the blue sides (7).

Note: The equivalence classes must be disjoined from each other (i.e. have no common elements), which is why zero factor homothecy is not allowed, otherwise the application of such a transformation would give each class a common element, the parallelogram reduced to one point. We remind in passing that such a parallelogram is not a sector because its orange side is zero.

\subsection{Definition of 2 Internal Composition Lawson the Sectoral Space}

\subsubsection{Addition of sectors}

The addition of 2 sectors consists of making the orange side (the reference) of the $2^{\text {nd }}$ sector coincide with the parallel of the orange side of the $1^{\text {st }}$ sector using transformations leaving the classes invariant (zoom, translation and rotation). For example, we can first make their orange side coincide by zooming, translating, rotating and secondly translate the $2^{\text {nd }}$ sector along the blue side of the $1^{\text {st }}$ sector so as to make the orange side of the $2^{\text {nd }}$ sector coincide on the side parallel to the orange side of the $1^{\text {st }}$. The resulting sector is then to redefine the blue side by connecting the blue ends (like adding vectors):

In Figure 4, (11) is obtained by positioning (9) on (8) and (12) is obtained by positioning (8) on (9). By the equivalence relation seen previously, (11) and (12) are equivalent. We show below that the addition is indeed commutative.

\subsubsection{Multiplication of Sectors}

The multiplication of 2 sectors consists in making the orange side of the $2^{\text {nd }}$ sector coincide with the blue side of the $1^{\text {st }}$ sector:

In Figure 5, (16) is obtained by positioning (14) on the blue side of (13) and (17) is obtained by positioning (13) on the blue side of (14). By the equivalence relation seen previously, (16) and (17) are equivalent. We show below that multiplication is indeed commutative.

\section{Algebraic Set of Pairs of Complex Vectors Equivalent to Sectors}

\subsection{Basic Algebraic Elements, Pairs of Complex Vectors}

All of these sectors can be noted very simply from the vector representation of complex numbers. As the previous representation suggests, these sectors are 

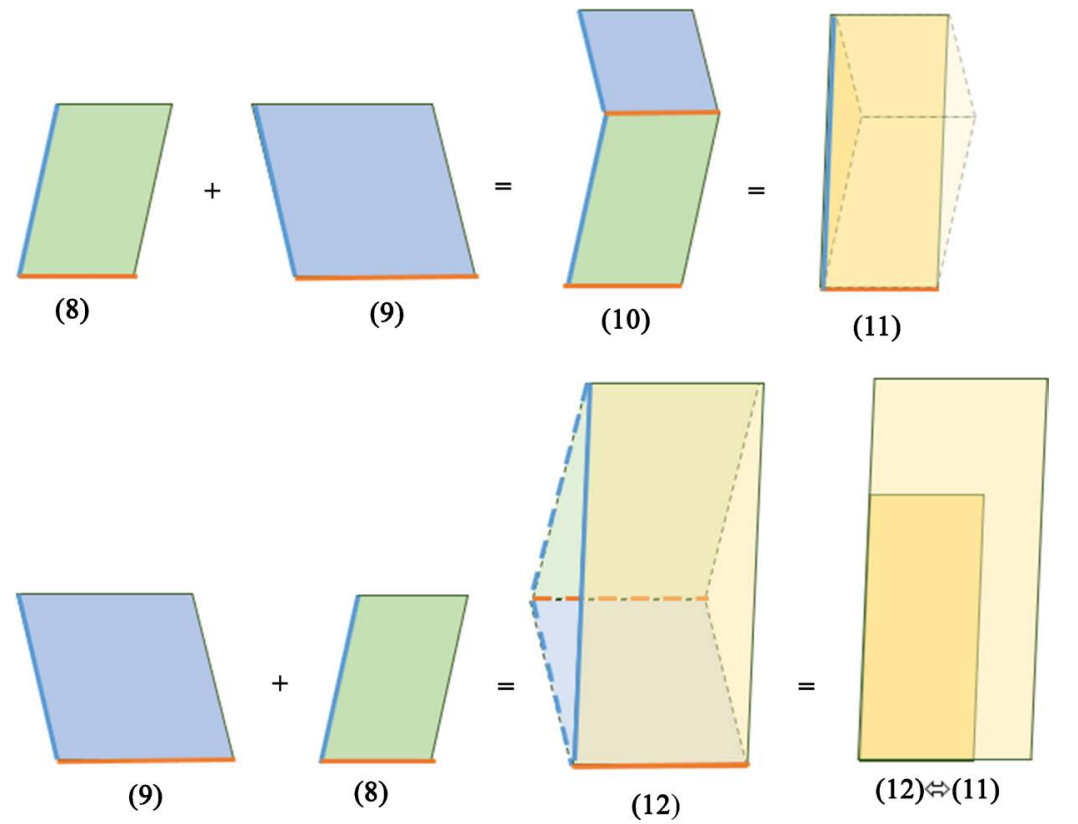

Figure 4. Addition of sectors.

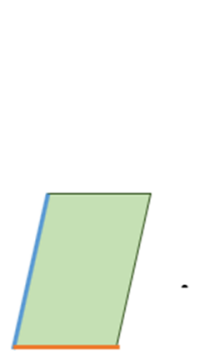

(13)

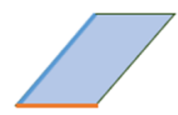

(14)

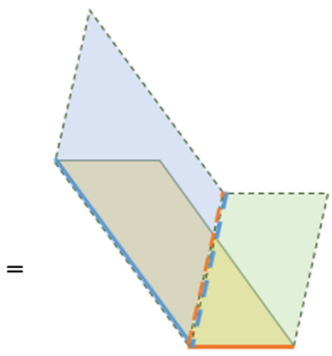

(15)

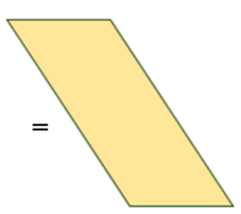

(16)

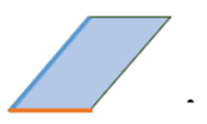

(14)

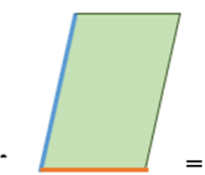

(13)

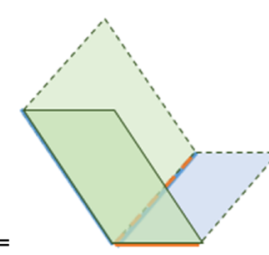

(17)

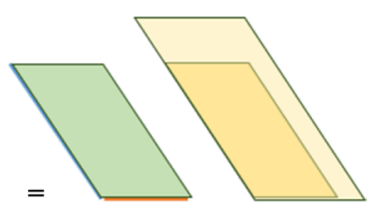

$(17) \Leftrightarrow(16)$

Figure 5. Multiplication of sectors.

characterized by their orange and blue sides. We can represent these 2 segments by vectors of complex space (in other words by 2 complex numbers). So if we denote $z_{1}$ and $z_{2}, 2$ complex numbers, any sector is defined as a 2 -tuple $\left(z_{1}, z_{2}\right)$. We can conventionally say that the $1^{\text {st }}$ member of the 2-tuple represents the orange side and the $2^{\text {nd }}$ the blue side(Figure 6).

In the geometric representation, we previously posed that the orange side should not be zero. We will make the same assumption by posing $z_{1} \neq 0$.

\subsection{Equivalence Classes on Pairs of Complex Vectors}

The equivalence class that we previously defined on the sectors corresponds to 


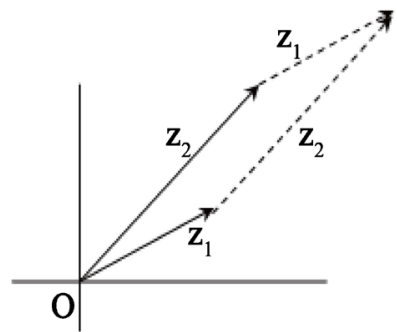

$\left(z_{1}, z_{2}\right)$

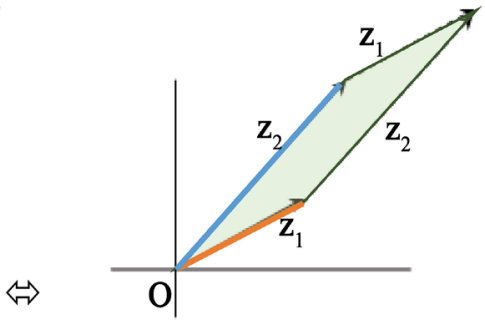

Sector (oriented parallelogram)

Figure 6. Sector and pair of complex vectors.

the following equivalence relation on the pairs of complexes:

$$
\left(z_{1}, z_{2}\right) \sim\left(z_{3}, z_{4}\right) \Leftrightarrow z_{2} \cdot z_{3}=z_{1} \cdot z_{4} \text { with } z_{1} \neq 0
$$

Let's check the equivalence of the classes of the sectors with the classes of these pairs of complexes. For sectors, the geometric transformations of the same equivalence class allow the 2 orange sides and the 2 blue sides of 2 sectors to correspond. In this new representation, we must therefore find the algebraic transformations to be able to transform one of the two 2-tuples so as to obtain both $z_{1}=z_{3}$ and $z_{2}=z_{4}$.

Because of the characteristics of the vector space of complex numbers, the transformations that apply to sectors (rotation and zoom) find their equivalence, for 2-tuples of complexes, in the simple multiplication by the same complex number of each of the components of the 2-tuple (so that the whole of the latter undergoes transformations without deformation). The complex number module acts as a zoom and the phase acts as a rotation. The translation is naturally ensured by the fact that the complexes are vectors. We thus define all these transformations (rotation and zoom) by the single relation (non-zero multiplication by $z_{3}$ of each component of the 2 -tuple):

$$
\left(z_{1}, z_{2}\right) \sim\left(z_{1} z_{3}, z_{2} z_{3}\right) \text { avec } z_{3} \neq 0
$$

We verify that this transformation is consistent with the equivalence relation:

$$
\left(z_{1}, z_{2}\right) \sim\left(z_{1} z_{3}, z_{2} z_{3}\right) \Leftrightarrow z_{2} z_{1} z_{3}=z_{1} z_{2} z_{3}
$$

This transformation does not modify the equivalence class, it represents the invariance of this space by translation, rotation and zoom.

Note: For the same reasons as for the geometric sectors, the multiplicative factor $z_{3}$ must not be zero otherwise the equivalence classes would no longer be disjoint (and would have the 2 -tuple $(0,0)$ as common representative and which is not an element of our space).

We note that if we match all the free sectors on the same origin (the origin of the complex plane in which we define the complex 2-tuples), we then have a bijection between these 2-tuples $\left(z_{1}, z_{2}\right)$ and the sectors linked to this origin. Given that in the 2 representations, algebraic and geometric, the equivalence classes identify elements in the same way, i.e. by a rotation and a zoom, the bijection is preserved between the sector classes and the complex 2-tuple classes. 


\subsection{Definition of 2 Internal Composition Laws for the Set of Pairs of Complexes}

We are going to define 2 internal composition laws (addition and multiplication) on the classes of pairs of complex vectors equivalent to the internal composition laws of sectors.

\subsubsection{Addition of Complex 2-Tuples}

We can translate the addition of sectors in algebraic form as follows (Figure 7):

$$
\left(z_{1}, z_{2}\right)+\left(z_{3}, z_{4}\right)=\left(z_{1} z_{3}, z_{2} z_{3}+z_{1} z_{4}\right)
$$

\subsubsection{Multiplication of Complex 2-Tuples}

We can translate the multiplication of sectors in algebraic form as follows (Figure 8)

$$
\left(z_{1}, z_{2}\right) \cdot\left(z_{3}, z_{4}\right)=\left(z_{1} z_{3}, z_{4} z_{2}\right)
$$

Note: The previous definitions of the classes and the 2 internal laws of these 2-tuples make it possible to see (via the algebraic representation) the sectors in the form of a fraction of complex numbers and thus to provide a possible notation for these sectors: “ $\left(z_{1}, z_{2}\right) \sim z_{2} / z_{1}$ ”. This point of view allows us to find the previous results:

$$
\frac{Z_{2}}{Z_{1}}+\frac{Z_{4}}{Z_{3}}=\frac{Z_{2} Z_{3}+z_{1} z_{4}}{Z_{1} Z_{3}} ; \frac{z_{2}}{Z_{1}} \cdot \frac{z_{4}}{Z_{3}}=\frac{Z_{2} z_{4}}{Z_{1} Z_{3}}
$$

\section{Definition of External Composition Laws on These Geometric and Algebraic Objects}

This algebraic representation by pair of complex numbers will allow us to

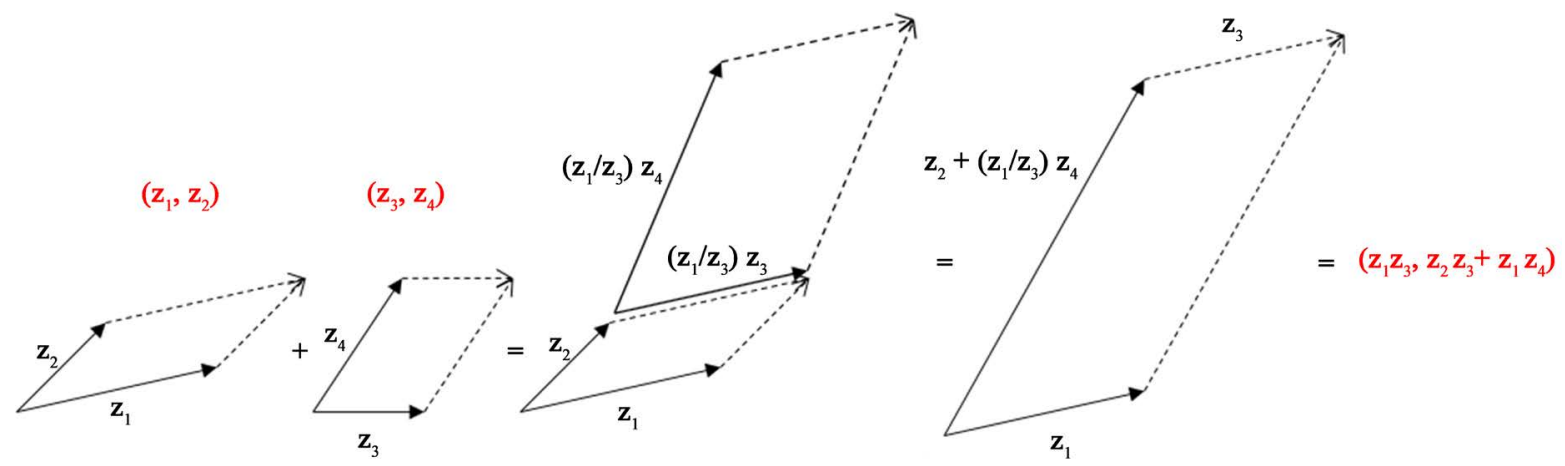

Figure 7. Addition of pairs of complexes.
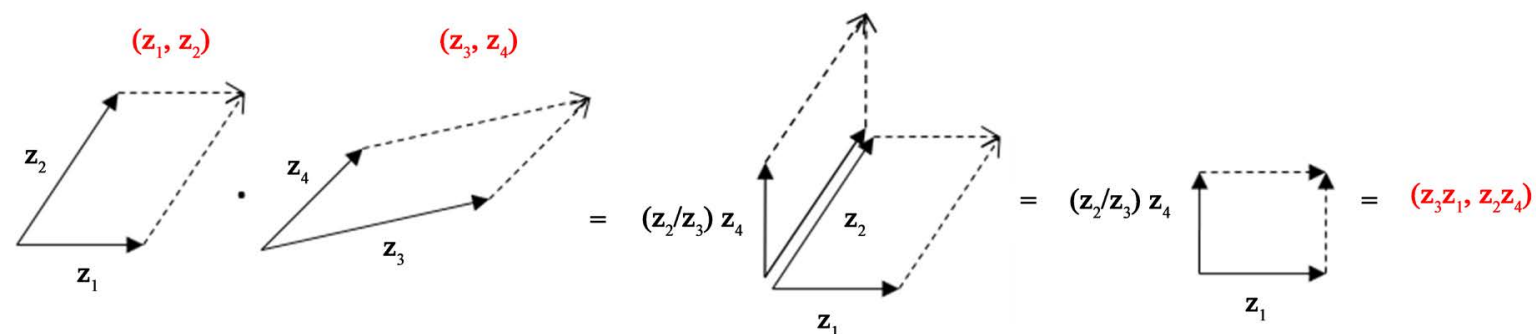

Figure 8. Multiplication of pairs of complexes. 
define 2 external composition laws (multiplication and exponentiation by a number) on these classes of objects. We will first geometrically introduce these external laws from the internal laws (addition and multiplication). We will then "naturally" extend these laws thanks to the algebraic representation of complex 2-tuples. In our study, the set of numbers, associated with our space, for these external composition laws will be the set of complex numbers.

\subsection{Multiplication of Sectors by a Number}

For the geometric representation: From the internal composition law (addition), we notice that adding $\mathrm{N}$ times the same sector consists in stacking the sectors on top of each other which ultimately corresponds to multiplying by $\mathrm{N}$ the blue side only(Figure 9).

For the algebraic representation: Using the "internal" addition of 2-tuples, we have:

$$
\left(z_{1}, z_{2}\right)+\left(z_{3}, z_{4}\right)=\left(z_{1} z_{3}, z_{2} z_{3}+z_{1} z_{4}\right)
$$

Either with $z_{1}=z_{3}$ et $z_{2}=z_{4}$

$$
\left(z_{1}, z_{2}\right)+\left(z_{1}, z_{2}\right)=2\left(z_{1}, z_{2}\right)=\left(z_{1} z_{1}, z_{2} z_{1}+z_{1} z_{2}\right)=\left(z_{1}, 2 z_{2}\right)
$$

We find the same result as for the geometric representation. Consequently, for the geometrical representation, the multiplication by a complex number $z_{3}$ will consist of multiplying the vector "blue side" by $z_{3}$. For the algebraic representation, the multiplication by a complex number $z_{3}$ will consist of multiplying the $2^{\text {nd }}$ member of the 2-tuple:

$$
z_{3}\left(z_{1}, z_{2}\right)=\left(z_{1}, z_{3} z_{2}\right)
$$

Note: We will note the consistency with the writing " $z_{2} / z_{1}$ " for which " $z_{2} / z_{1}+z_{2} / z_{1}=\left(2 z_{2}\right) / z_{1}$ ” or even " $k\left(z_{2} / z_{1}\right)=\left(k z_{2}\right) / z_{1}$ ”.

\subsection{Exponentiation of Sectors by a Number}

For the geometric representation: From the law of internal composition (multiplication), we notice that the fact of multiplying $\mathrm{N}$ times the same object consists in putting the sectors next to each other (by applying the orange reference on the blue side in a construction in spiral) which ultimately corresponds to raise to the
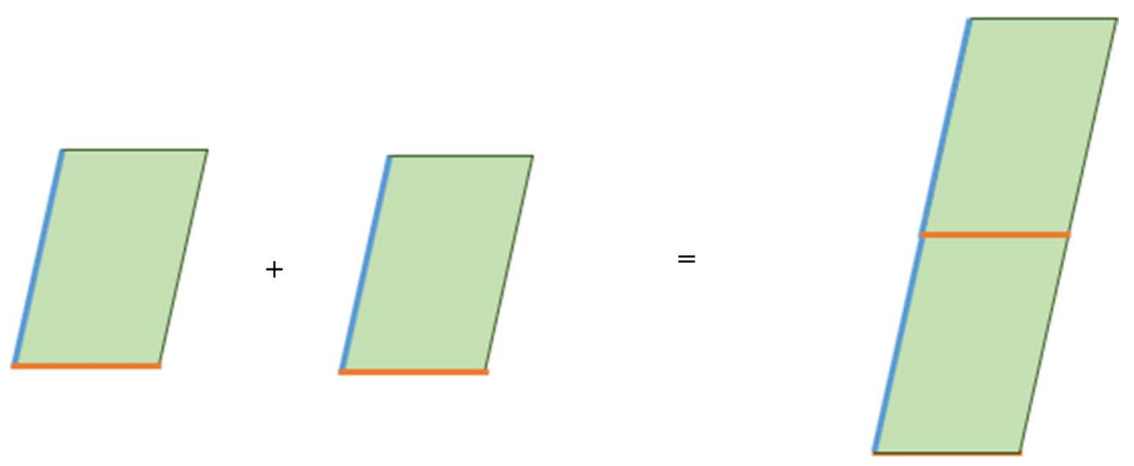

Figure 9. From sectoral addition to multiplication of sectors by a number. 
power of $\mathrm{N}$ both the orange side and the blue side (using the invariance by zoom), as shown in Figure 10.

For the algebraic representation: By taking up the multiplication of 2-tuples, we have

$$
\left(z_{1}, z_{2}\right) \cdot\left(z_{3}, z_{4}\right)=\left(z_{1} z_{3}, z_{4} z_{2}\right)
$$

Either with $z_{1}=z_{3}$ et $z_{2}=z_{4}$

$$
\left(z_{1}, z_{2}\right) \cdot\left(z_{1}, z_{2}\right)=\left(z_{1}, z_{2}\right)^{2}=\left(z_{1} z_{1}, z_{2} z_{2}\right)=\left(z_{1}^{2}, z_{2}^{2}\right)
$$

We find the same result as for the geometric representation. Consequently, for the geometric representation, the exponentiation by a complex number $z_{3}$ will consist in raising to the power of $z_{3}$ both the complex number representing the "blue side" and the complex number representing the "orange side". For the algebraic representation, the exponentiation by a complex number $z_{3}$ will consist in raising to the power of $z_{3}$ both the 2 members of the 2-tuple:

$$
\left(z_{1}, z_{2}\right)^{z_{3}}=\left(z_{1}^{z_{3}}, z_{2}^{z_{3}}\right)
$$

Note: We will note the consistency with the writing " $z_{2} / z_{1}$ ” for which “ $\left(z_{2} / z_{1}\right)\left(z_{2} / z_{1}\right)=z_{2}^{2} / z_{1}^{2}$ ” or even " $\left(z_{2} / z_{1}\right)^{k}=z_{2}^{k} / z_{1}^{k}$ ”.

\section{Properties of a Sector Structure}

At this stage, we have therefore developed a sectoral space in which we have defined each constituent element, the free sectors, in a geometric manner (parallelogram seen as bivectors of the complex plane) and in an algebraic manner (2-tuple of complex number like " $x / y$ "). In these 2 representations we have defined 2 internal composition laws (addition and multiplication) and 2 external composition laws (multiplication and exponentiation). We will now study the structuring properties of these laws of composition. In order to simplify this study for the geometric representation, we will introduce the notion of linked sector.
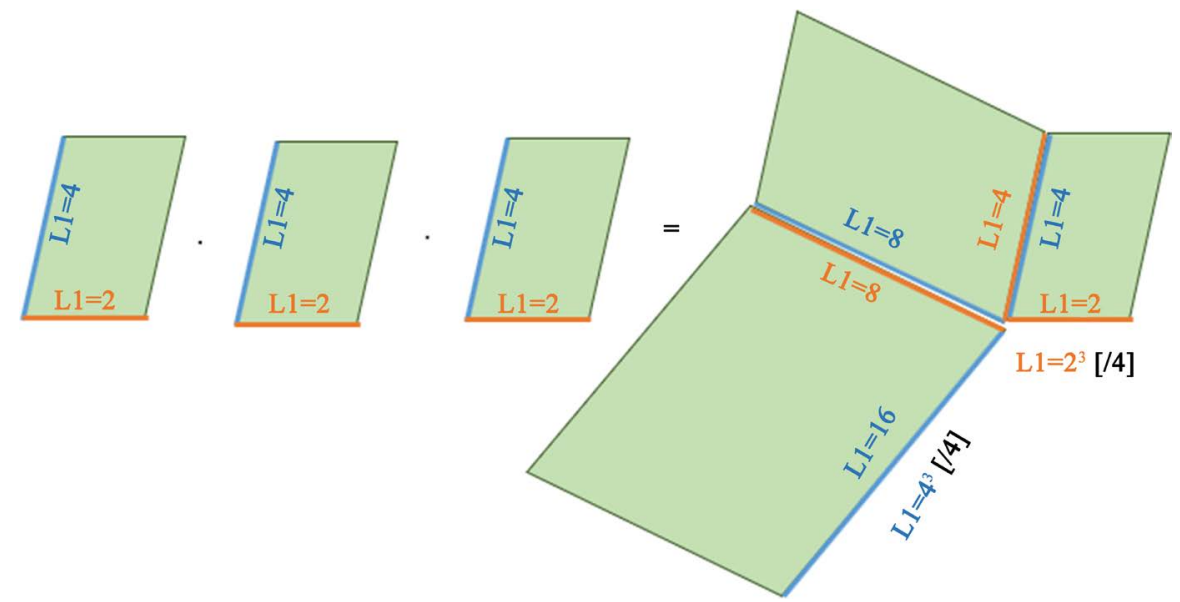

Figure 10. From sectoral multiplication to exponentiation of sectors by a number. 


\subsection{Sector Structure}

Linked sectors (on the same origin and the same reference side):

To effectively compare 2 sectors, it is interesting to make them share common elements (origin, orientation and size). And even more generally, it is interesting that all the sectors are comparable in relation to these same common elements. Concretely, a procedure must be found to find the unique representatives of each class who share these common elements (origin, orientation and size). Like the transition from free vectors to vectors linked to the same reference origin (fixing a basic position common to all) [1], we are going to pass from free sectors to sectors linked to the same reference origin (fixing a basic position common to all) and on the same reference side (setting a basic orientation and size common to all). According to the equivalence relation of the sectors, two sectors which are only differentiated by a translation represent the same class. So, let's take the point at the intersection of the orange side and the blue side as a reference and translate all the sectors on the same arbitrary point. The sectors that were differentiated by only a translation, no longer form a single sector (each overlapping this representative). Let's do the same procedure for the 2 other transformations leaving the sectors of the same class invariant (rotation and zoom). According to the equivalence relation of the sectors, two sectors which differ only by rotation represent the same class. So, let's take the orange side as the reference direction and rotate all the sectors so that their orange side is all oriented in the same way. The sectors that were differentiated by only a rotation, no longer form a single sector (each overlapping this representative). Finally, according to the equivalence relation of the sectors, two sectors which differ only by a homothety (of non-zero factor) represent the same class. Then let's take an orange side of unit length as a reference and apply an homothety to all the sectors so that their orange side exactly overlaps. The sectors which were differentiated by only an homothety, no longer form a single sector (each overlapping this representative). Thus, by this procedure (choice of an origin and a reference side) we define unique representatives of each equivalence class. We will henceforth place ourselves in this space of "sectors linked" to an origin and to a unit side (which will be the orange side). This step will simplify the study of the geometric operations of the sectoral space because now we can place ourselves in a 2-dimensional vector space in which we choose an orthonormal base. We will place all previous unique representatives on the origin with the orange side on the vector of the $1^{\text {st }}$ component of the base. All sectors will be distinguished by their blue side only (which will be a vector of this vector space). Conversely, any vector $\mathrm{V}$ of the vector space will correspond to a single sector, for which the vector $\mathrm{V}$ is on the blue side and the $1^{\text {st }}$ vector of the base of the vector space on the orange side. We thus have a bijection between the vector space of complex numbers and the elements of sector space. It is this space which will henceforth be named sectoral space. In 2-tuple representation, this would lead to defining the 2-tuple $(1, z)$ as the representative of each class with 
$z$ a complex number. But the algebraic manipulation of 2-tuples being as easy with the single representative as with a generic representative $\left(z_{1}, z_{2}\right)$ that we will continue to use the generic 2-tuple in the algebraic representation of the sectors.

\subsection{Properties of the Internal Composition Laws of Sectors}

We will now establish the properties of the different composition laws defined previously. We will demonstrate them in the 2 representations, sectors of sectoral space and pairs of complex numbers. We will denote " $S 1$ ", " $S 2$ " ... the sectors and " $\left(z_{1}, z_{2}\right)$ " the pairs of complexes.

\subsubsection{Sectoral Addition}

\section{Commutativity}

For the sector space: Addition involves translating one of the sectors from the origin to match the orange side on the end of the other. With the definition of the previous linked sectors (same origin and same reference orange coast), this operation is reduced to the vector addition on the blue sides of the sectors. As vector addition is commutative [1], sector addition is also commutative (Figure 11)

$$
S 1+S 2=S 2+S 1
$$

For the algebraic representation of 2-tuples of complex numbers (with the properties of complex numbers [2])

$$
\left(z_{1}, z_{2}\right)+\left(z_{3}, z_{4}\right)=\left(z_{1} z_{3}, z_{2} z_{3}+z_{1} z_{4}\right)=\left(z_{3} z_{1}, z_{4} z_{1}+z_{3} z_{2}\right)=\left(z_{3}, z_{4}\right)+\left(z_{1}, z_{2}\right)
$$

\section{Associativity}

For the sector space: Since the addition of the linked sectors is reduced to the vector addition on the blue sides of the sectors, the vector addition being associative, the sector addition is also

$$
(S 1+S 2)+S 3=S 1+(S 2+S 3)
$$

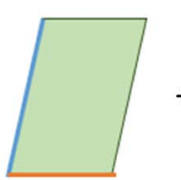

$S 1$

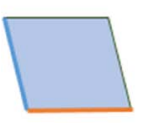

$S 2$
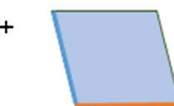

$S 2$

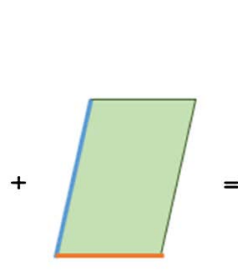

$S 1$

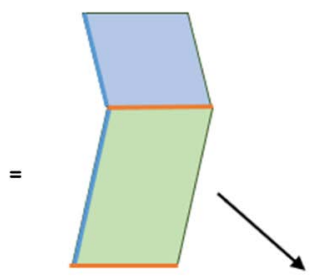

$S 1+S 2$

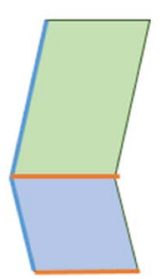

$s 2+s 1$

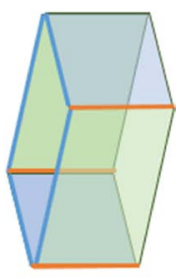

Figure 11. Commutativity of sectoral addition. 
For the algebraic representation of 2-tuples of complex numbers

$$
\begin{aligned}
\left(\left(z_{1}, z_{2}\right)+\left(z_{3}, z_{4}\right)\right)+\left(z_{5}, z_{6}\right) & =\left(z_{1} z_{3}, z_{2} z_{3}+z_{1} z_{4}\right)+\left(z_{5}, z_{6}\right) \\
& =\left(\left(z_{1} z_{3}\right) z_{5},\left(z_{2} z_{3}+z_{1} z_{4}\right) z_{5}+\left(z_{1} z_{3}\right) z_{6}\right) \\
\left(z_{1}, z_{2}\right)+\left(\left(z_{3}, z_{4}\right)+\left(z_{5}, z_{6}\right)\right) & =\left(z_{1}, z_{2}\right)+\left(z_{3} z_{5}, z_{4} z_{5}+z_{3} z_{6}\right) \\
& =\left(z_{1}\left(z_{3} z_{5}\right), z_{2}\left(z_{3} z_{5}\right)+z_{1}\left(z_{4} z_{5}+z_{3} z_{6}\right)\right)
\end{aligned}
$$

The associativity and distributivity of complex numbers (which makes up each component of the 2-tuple) gives us

$$
\left(\left(z_{1}, z_{2}\right)+\left(z_{3}, z_{4}\right)\right)+\left(z_{5}, z_{6}\right)=\left(z_{1}, z_{2}\right)+\left(\left(z_{3}, z_{4}\right)+\left(z_{5}, z_{6}\right)\right)
$$

\section{Neutral element}

For the sector space: Let's take the zero blue side sector that we will name the zero sector (Figure 12).

Note on representation: Theoretically only the orange side should be seen, but to make the operations more explicit, we slightly shift the opposite side (black) in this representation (lines on vertical sides are reduced to points but we have shown only the blue side, necessary for operations).

It represents the neutral element of the sector addition (Figure 13) because this operation is reduced to the vector addition on the blue side and the latter is zero in the case of the zero sector.

As usual, it will be noted $0: 0+S 1=S 1+0=S 1$

For the algebraic representation of pairs of complex numbers, this element is noted $(z, 0) \sim(1,0)$ and we verify

$$
\begin{aligned}
& \left(z_{1}, z_{2}\right)+(z, 0)=\left(z_{1} z, z_{2} z+z_{1} 0\right)=\left(z_{1} z, z_{2} z\right) \sim\left(z_{1}, z_{2}\right) \\
& (z, 0)+\left(z_{1}, z_{2}\right)=\left(z z_{1}, 0 z_{1}+z z_{2}\right)=\left(z z_{1}, z z_{2}\right) \sim\left(z_{1}, z_{2}\right)
\end{aligned}
$$

Opposite

For the sector space: The sectoral addition being reduced to a vector addition

Zero sector $=\langle 0 »$

Figure 12. Zero sector.

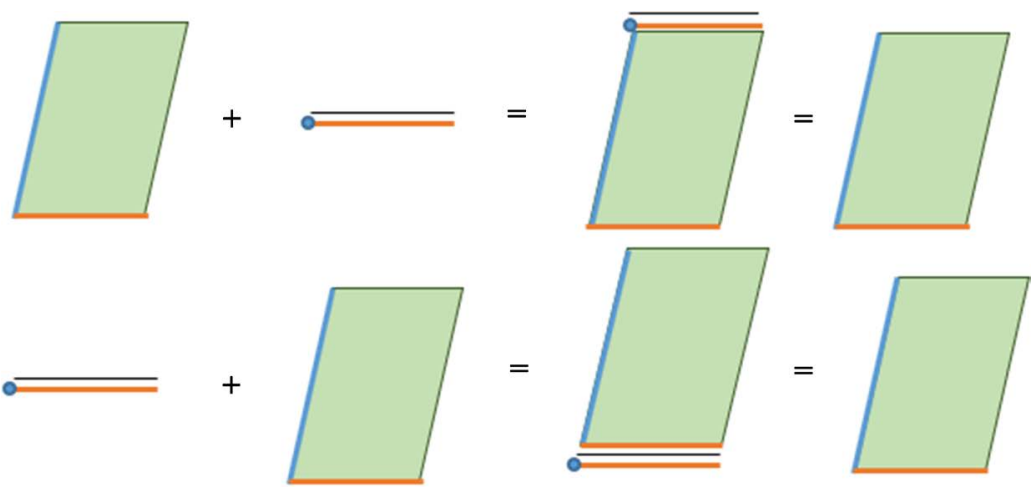

Figure 13. Neutral element of sectoral addition. 
on the blue sides(Figure 14), we deduce that the opposite element is the sector defined by the opposite vector on the blue side (always with the orange side on the $1^{\text {st }}$ vector base unit). As usual, it will be noted “ $-S 1$ ”.

For the algebraic representation of 2-tuples of complex numbers, the opposite of $\left(z_{1}, z_{2}\right)$ is the element $\left(z_{1},-z_{2}\right)$ and we verify

$$
\left(z_{1}, z_{2}\right)+\left(z_{1},-z_{2}\right)=\left(z_{1} z_{1}, z_{2} z_{1}-z_{1} z_{2}\right) \sim\left(z_{1}, z_{2}-z_{2}\right)=\left(z_{1}, 0\right) \sim(1,0)
$$

And vice versa. According to the multiplication by a number, this element can also be written

$$
\left(z_{1},-z_{2}\right)=-\left(z_{1}, z_{2}\right)
$$

\subsubsection{Sectoral Multiplication}

\section{Commutativity}

For the sector space: Multiplication consists of joining the orange side of one sector to the blue side of the other sector. This is achieved by turning it and then making it undergo a homothety so that its orange side exactly matches the blue side of the other. The resulting sector (S3 in Figure 15) is then formed on the initial orange unit side of the sector which has not been transformed and on the new blue side (turned and zoomed) of the other transformed sector.

Note that the sector is generally identified by 2 components: the angle (between the orange side and the blue side) and the length of the blue side. In our 2D space, these 2 components are reals. The multiplication is then broken down into 2 "sub-operations" on the sector components, an addition of the angle (orange side-blue side) of each sector and a multiplication of the length of the blue side of each sector (because the side orange being of unit length, its transfer on the blue side implies a zoom of a factor equal to the length of the blue side of the other sector). These remarks allow us to show the commutativity of sectoral multiplication. Indeed, the addition of the angles (of the reals) being

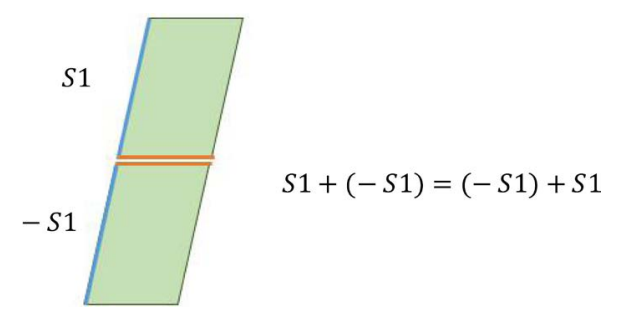

Figure 14. Opposite sector of sectoral addition.

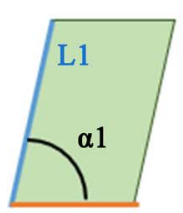

$S 1$

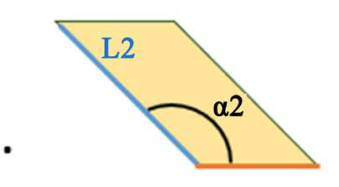

S2

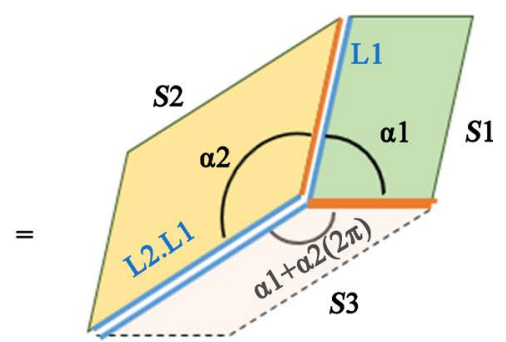

Figure 15. Sectoral multiplication as angles' addition and lengths' multiplication. 
commutative, it will therefore be identical by interchanging the 2 sectors. This also means that in the 2 cases ( $S 1 \cdot S 2$ or $S 2 \cdot S 1$ ) the blue side will have the same direction and same orientation. It then remains to verify that, in both cases, their length remains the same. This length will be $L 1 \cdot L 2$ in one case and $L 2 \cdot L 1$ in the other. However, the multiplication of these 2 (real) lengths is commutative, $L 1 \cdot L 2=L 2 \cdot L 1$, which demonstrates the equality of the lengths of the blue sides in the 2 cases of multiplication. We conclude that the multiplication of sectors is commutative.

$$
S 1 \cdot S 2=S 2 \cdot S 1
$$

For the algebraic representation of 2-tuples of complex numbers

$$
\left(z_{1}, z_{2}\right) \cdot\left(z_{3}, z_{4}\right)=\left(z_{1} z_{3}, z_{4} z_{2}\right)=\left(z_{3} z_{1}, z_{2} z_{4}\right)=\left(z_{3}, z_{4}\right) \cdot\left(z_{1}, z_{2}\right)
$$

\section{Associativity}

For the sector space: As with commutativity, multiplication resulting in the addition of angles and multiplication of lengths, associativity is ensured by the associativity of addition (angles) and the associativity of multiplication (lengths).

$$
(S 1 \cdot S 2) \cdot S 3=S 1 \cdot(S 2 \cdot S 3)
$$

For the algebraic representation of 2-tuples of complex numbers

$$
\begin{aligned}
& \left(\left(z_{1}, z_{2}\right) \cdot\left(z_{3}, z_{4}\right)\right) \cdot\left(z_{5}, z_{6}\right)=\left(z_{1} z_{3}, z_{4} z_{2}\right) \cdot\left(z_{5}, z_{6}\right)=\left(\left(z_{1} z_{3}\right) z_{5}, z_{6}\left(z_{4} z_{2}\right)\right) \\
& \left(z_{1}, z_{2}\right) \cdot\left(\left(z_{3}, z_{4}\right) \cdot\left(z_{5}, z_{6}\right)\right)=\left(z_{1}, z_{2}\right) \cdot\left(z_{3} z_{5}, z_{6} z_{4}\right)=\left(z_{1}\left(z_{3} z_{5}\right),\left(z_{6} z_{4}\right) z_{2}\right)
\end{aligned}
$$

The associativity of complex numbers (which makes up each component of the 2-tuple) gives us

$$
\left(\left(z_{1}, z_{2}\right) \cdot\left(z_{3}, z_{4}\right)\right) \cdot\left(z_{5}, z_{6}\right)=\left(z_{1}, z_{2}\right) \cdot\left(\left(z_{3}, z_{4}\right) \cdot\left(z_{5}, z_{6}\right)\right)
$$

\section{Neutral element}

For the sector space: Let's take the blue side sector equal to the orange side that we will name the flat unit sector or more simply the unit sector(Figure 16).

Note on the representation: Theoretically, the orange and blue sides overlap but for reasons of clarity, we slightly shift the 2 sides in this visual representation (we have not put a line on the vertical sides because they do not exist).

It represents the neutral element of sectoral multiplication (Figure 17) because this operation then results in identically overlapping one or the other of the sides (depending on the direction of the multiplication).

As usual, we note it $1: 1 \cdot S 1=S 1 \cdot 1=S 1$

For the algebraic representation of 2-tuples of complex numbers, this element is noted $(z, z) \sim(1,1)$ and we verify

$$
\left(z_{1}, z_{2}\right) \cdot(z, z)=\left(z_{1} z, z z_{2}\right) \sim\left(z_{1}, z_{2}\right)
$$

Flat unit sector $=\langle 1 »$

Figure 16. Flat unit sector. 


$$
(z, z) \cdot\left(z_{1}, z_{2}\right)=\left(z z_{1}, z_{2} z\right) \sim\left(z_{1}, z_{2}\right)
$$

\section{Inverse}

For the sector space: The multiplication resulting to the addition of angles and the multiplication of lengths, the inverse sector is defined by the opposite of the angle and the inverse of the length (Figure 18). As is usual, it will be noted " $S 1^{-1}$ ".

For the algebraic representation of 2-tuples of complex numbers, the inverse of $\left(z_{1}, z_{2}\right)$ is the 2-tuple $\left(z_{1}^{-1}, z_{2}^{-1}\right)$ and we verify

$$
\left(z_{1}, z_{2}\right) \cdot\left(z_{1}^{-1}, z_{2}^{-1}\right)=\left(z_{1} z_{1}^{-1}, z_{2}^{-1} z_{2}\right)=(1,1)
$$

And vice versa. According to the exponentiation by a number, this element can also be written

$$
\left(z_{1}^{-1}, z_{2}^{-1}\right)=\left(z_{1}, z_{2}\right)^{-1}
$$

Notes: As usual for multiplicative groups, the inverse element of the null element $\left(z_{1}, 0\right)$ does not exist. Let's remind that by definition, we always have $z_{1} \neq 0$ in our set. But just let's look at the special case of multiplication by this null element. In the case of algebraic 2-tuples we have no problem, it is even an absorbing element:

$$
\begin{aligned}
& \left(z_{1}, 0\right) \cdot\left(z_{3}, z_{4}\right)=\left(z_{1} z_{3}, 0\right)=0 \\
& \left(z_{3}, z_{4}\right) \cdot\left(z_{1}, 0\right)=\left(z_{3} z_{1}, 0\right)=0
\end{aligned}
$$

In the geometrical case, the multiplication on the right by the null sector (Figure 19) does not pose either problem, it is also an absorbing element

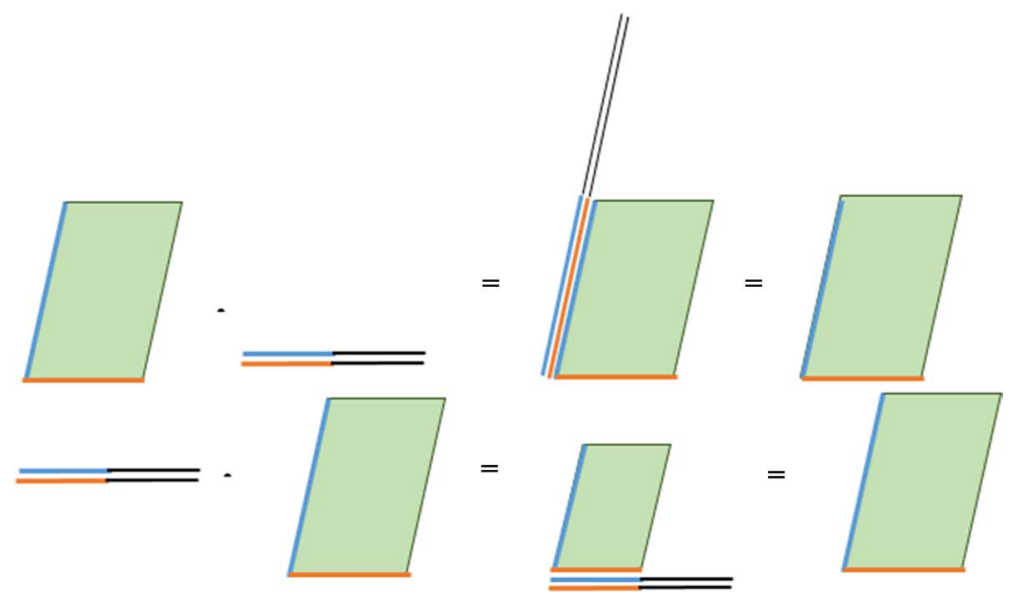

Figure 17. Neutral element of sectoral multiplication.

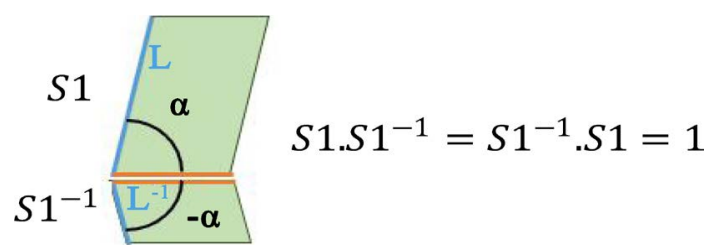

Figure 18. Inverse sector of sectoral multiplication. 


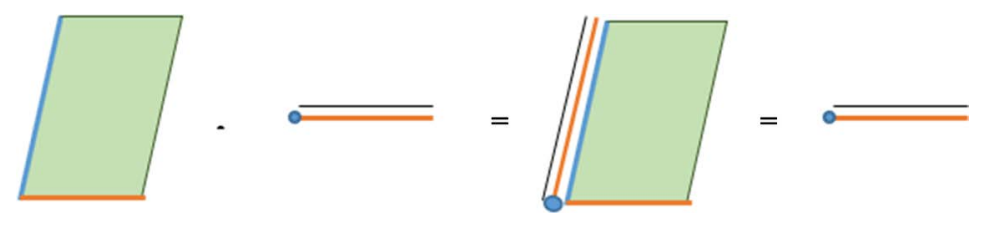

Figure 19. Multiplication on the right by the null sector.

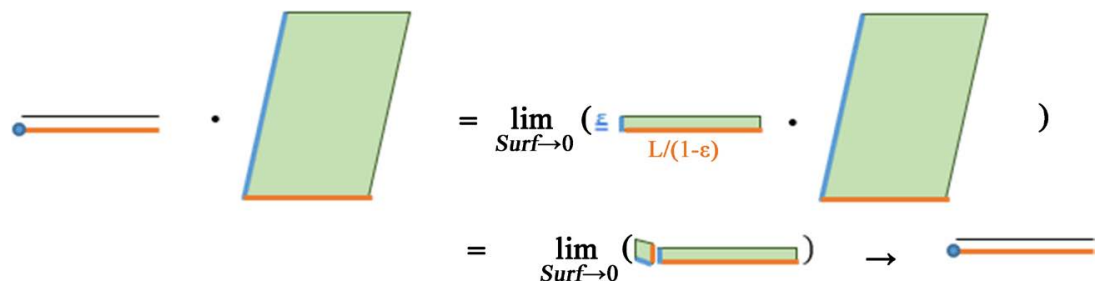

Figure 20. Multiplication on the left by the null sector.

On the other hand, the multiplication on the left by the null sector (Figure 20 ), in the geometrical case, cannot be expressed explicitly because one should transiently reduce the second member by a homothety of null factor what one excluded in the definition of the classes of equivalence. But we can give at least 5 ways to justify/extend the result of this operation:

1) As a limit case (by giving for the null sector a length $\varepsilon<1$ on the blue side and a length $L /(1-\varepsilon)$ on the orange side, $L$ being the length of the reference when the blue side is zero, then in causing $\varepsilon$ to tend towards 0 ), this operation remains consistent:

2) Extension of the commutativity: In order to maintain the commutativity of the sectoral multiplication, one can assume without contradiction that the multiplication on the left by the null sector gives the null sector.

3) The bijection of the sectors with the algebraic 2-tuples also makes it possible to justify the result of the multiplication on the left by the null sector without contradiction.

4) Extension of the absorbing element: The fact that this sector is an absorbing element for all the other cases of multiplication makes it possible to extend this result to the multiplication on the left by the null sector without contradiction.

5) From the distributivity of the multiplication over the addition (which we justify immediately after), we have on the one hand $S 1 \cdot S 2-S 1 \cdot S 2=(S 1-S 1) \cdot S 2$ and on the other hand $S 1 \cdot S 2-S 1 \cdot S 2=0$ from which we deduce that (S1$S 1) \cdot S 2=0$ which gives us $0 \cdot S 2=0$. Caution, " 0 " here represents the null sector and not the null complex number (relation then concerning the external multiplication which will be demonstrated below).

\subsubsection{Distributivity of Sectoral Multiplication over Sectoral Addition}

Exceptionally, we will start with the algebraic representation of pairs of complex numbers. Distributivity gives

$$
\begin{aligned}
\left(z_{1}, z_{2}\right) \cdot\left(\left(z_{3}, z_{4}\right)+\left(z_{5}, z_{6}\right)\right) & =\left(z_{1}, z_{2}\right) \cdot\left(z_{3} z_{5}, z_{4} z_{5}+z_{3} z_{6}\right) \\
& =\left(z_{1} z_{3} z_{5},\left(z_{4} z_{5}+z_{3} z_{6}\right) z_{2}\right)
\end{aligned}
$$




$$
\begin{aligned}
\left(\left(z_{3}, z_{4}\right)+\left(z_{5}, z_{6}\right)\right) \cdot\left(z_{1}, z_{2}\right) & =\left(z_{3} z_{5}, z_{4} z_{5}+z_{3} z_{6}\right) \cdot\left(z_{1}, z_{2}\right) \\
& =\left(z_{3} z_{5} z_{1}, z_{2}\left(z_{4} z_{5}+z_{3} z_{6}\right)\right) \\
\left(z_{1}, z_{2}\right) \cdot\left(z_{3}, z_{4}\right)+\left(z_{1}, z_{2}\right) \cdot\left(z_{5}, z_{6}\right) & =\left(z_{1} z_{3}, z_{4} z_{2}\right)+\left(z_{1} z_{5}, z_{6} z_{2}\right) \\
& =\left(z_{1} z_{3} z_{1} z_{5}, z_{4} z_{2} z_{1} z_{5}+z_{1} z_{3} z_{6} z_{2}\right)
\end{aligned}
$$

In this last relation, by dividing each component by non-zero $z_{1}$, and for the 2 other relations thanks to the distributivity of the complex numbers (which composes each component of the 2-tuples), one verifies that

$$
\begin{aligned}
\left(z_{1}, z_{2}\right) \cdot\left(\left(z_{3}, z_{4}\right)+\left(z_{5}, z_{6}\right)\right) & =\left(\left(z_{3}, z_{4}\right)+\left(z_{5}, z_{6}\right)\right) \cdot\left(z_{1}, z_{2}\right) \\
& =\left(z_{1}, z_{2}\right) \cdot\left(z_{3}, z_{4}\right)+\left(z_{1}, z_{2}\right) \cdot\left(z_{5}, z_{6}\right)
\end{aligned}
$$

For the sector space: Previously, we easily showed the properties of sectoral addition and sectoral multiplication independently by two different procedures. For the sectoral addition, it was the vector addition of the blue sides. For sectoral multiplication, it was both the addition of angles and the multiplication of lengths. For distributivity, the combination of these operations makes it more difficult to explain by these 2 methods. This difficulty allows us to set up a $3^{\text {rd }}$ "analytical" representation of the sectors which, in a way, makes the link between the geometric and algebraic representations of the sectors. Indeed, on the one hand the sectors of the sectoral space are explicitly defined and differentiated by the blue side only (and implicitly by the origin and the orange side unit but which are the same for all the sectors) which forms, as we have already seen, a bijection between the vector space of complex numbers and the sector space. This blue side being represented by a complex vector, a sector can therefore be

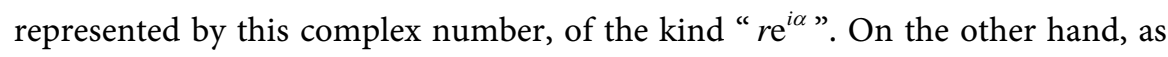
we saw previously, the sectors of the sectoral space (linked to the origin and to the reference side) are, in the form of a 2 -tuple, the elements $(1, z)$. These 2 -tuples being equivalent to the rational notation " $z / 1=z$ ", again, we deduce the expression of the sector in its exponential form of kind " $r \mathrm{e}^{\mathrm{i} \alpha}$ ". The structure we are studying is associated with the set of complex numbers. But we can certainly associate such a sectoral space with other sets of numbers, on the only condition that these numbers satisfy the rules of the laws of composition of this space. For example, it is likely that the quaternions could be a set associated with

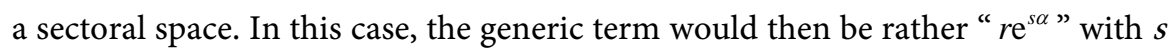
a pure imaginary unitary quaternion. For this, we will not note the sectors explicitly in the form " $r \mathrm{e}^{i \alpha}$ ", but in a more general form:

$$
S 1 \sim R 1 X^{A 1}
$$

This writing maintains the definitions and properties of the composition laws already observed before and they will follow the rules expected by the sectors. We also retrieve by this notation, that the sectoral multiplication is equivalent to the addition of angles and the multiplication of lengths, since

$$
S 1 \cdot S 2 \sim R 1 X^{A 1} \cdot R 2 X^{A 2}=R 1 R 2 X^{A 1+A 2}
$$

Thus, the algebra of sectors turns out to be equivalent to that of complex 
numbers (for the complex case we have $X \sim \mathrm{e}^{i}$ ). The distributivity is then ensured by the distributivity of the complexes

$$
S 1 \cdot(S 2+S 3)=S 1 \cdot S 2+S 1 \cdot S 3=S 2 \cdot S 1+S 3 \cdot S 1=(S 2+S 3) \cdot S 1
$$

But more generally (if we don't limit ourselves to the algebra of complexes)

$$
\begin{aligned}
& S 1 \cdot(S 2+S 3) \sim R 1 X^{A 1} \cdot\left(R 2 X^{A 2}+R 3 X^{A 3}\right) \\
& =R 1 R 2 X^{A 1+A 2}+R 1 R 3 X^{A 1+A 3} \sim S 1 \cdot S 2+S 1 \cdot S 3
\end{aligned}
$$

In the end, the 2 internal composition laws of the sectoral space form a field.

\subsection{Properties of the External Composition Laws of Sectors}

We can see the integration of external laws into these structures as "powerful" tools allowing us to extend the manipulation of objects in space by an internal operation which is not defined. Thus, in vector spaces, the rules put in place make it possible to integrate a form of multiplication which is not defined between the vectors but which can be applied between these vectors by an addressing consistent with a set of numbers handling this operation. In the sector space we started the same procedure, with the external multiplication consistent with the internal addition, but especially with the external exponentiation consistent with the internal multiplication that is quite new. But in order to finalize the consistency of this structure, we must therefore set up coherent addressing rules between these sectors and this set of numbers associated with this space (compatibility of internal and external operations). In the following, we will abundantly use the particularity of complex numbers which, on the one hand, are vectors (and therefore follow the properties of a vector space) but, on the other hand, are objects with richer properties such as " $z^{z_{1}+z_{2}}=z^{z_{1}} z^{z_{2}}$ " or else " $\left(z_{1} z_{2}\right)^{z}=z_{1}^{z} z_{2}^{z}$ ". In particular, following what we have just seen (bijection of the sectors with complex vectors via the blue side of the sector), the blue side will be explicitly seen as a complex vector.

\subsubsection{Multiplication of Sectors by a Number}

Let's show that we have: $0 \cdot S 1=0$

Reminder: In this expression, this is external multiplication, unlike the similar writing mentioned above and which concerned sectoral multiplication (internal composition law).

For the sector space: Our definition of multiplication by a number consists in multiplying the "blue side" vector by this same number. In our case, it consists in multiplying by zero the blue side, which gives a sector of blue side of zero length, that is to say the zero-sector noted 0 . The relation is thus verified. We will note the consistency with the exponential notation " $0 \cdot R 1 X^{A 1}=0$ ".

For the algebraic representation of 2-tuples of complex numbers, the definition of multiplication by a number gives

$$
0\left(z_{1}, z_{2}\right)=\left(z_{1}, 0 z_{2}\right)=\left(z_{1}, 0\right)=0
$$

Which verifies the relationship. 
Let's show that we have: $1 \cdot S 1=S 1$

For the sector space: Our definition of multiplication by a number consists in multiplying the "blue side" vector by this same number. In our case, it consists in multiplying by 1 the blue side, which gives an identical blue side sector, that is to say that the sector is unchanged. The relationship is therefore verified. We'll note the consistency with the exponential notation " $1 \cdot R 1 X^{A 1}=R 1 X^{A 1}$ ”.

For the algebraic representation of 2-tuples of complex numbers, the definition of multiplication by a number gives:

$$
1\left(z_{1}, z_{2}\right)=\left(z_{1}, 1 z_{2}\right)=\left(z_{1}, z_{2}\right)
$$

Which verifies the relationship.

Let's show that we have: $z_{1}\left(z_{2} S 1\right)=\left(z_{1} z_{2}\right) S 1$

For the sector space: Our definition of multiplication by a number consists in multiplying the "blue side" vector by this same number, in this case " $z_{2}$ ". Once this multiplication has been carried out, it consists in multiplying this new "blue side" vector by the second number, " $z_{1}$ ", which finally consists in directly multiplying the initial "blue side" vector by the number " $z_{1} z_{2}$ ". It actually results from the multiplication of a vector (our blue side) by a number which is a property verified by any vector. We will note the consistency with the exponential notation " $z_{1}\left(z_{2} R 1 X^{A 1}\right)=\left(z_{1} z_{2} R 1 X^{A 1}\right)=\left(z_{1} z_{2}\right) R 1 X^{A 1}$ ”.

For the algebraic representation of 2-tuples of complex numbers, the definition of multiplication by a number gives:

$$
z_{4}\left(z_{3}\left(z_{1}, z_{2}\right)\right)=z_{4}\left(z_{1}, z_{3} z_{2}\right)=\left(z_{1}, z_{4}\left(z_{3} z_{2}\right)\right)=\left(z_{1},\left(z_{4} z_{3}\right) z_{2}\right)=\left(z_{4} z_{3}\right)\left(z_{1}, z_{2}\right)
$$

Which verifies the relationship.

Let's show that we have: $\left(z_{1}+z_{2}\right) S 1=z_{1} S 1+z_{2} S 1$

For the sector space: Our definition of multiplication by a number consists in multiplying the "blue side" vector by this same number, in this case " $z_{1}+z_{2}$ ". Once this multiplication has been carried out, it comes from the properties of the multiplication of a vector (our blue side) by a number, that our blue side is the sum of 2 "blue side" vectors. According to the definition of multiplication seen above, we can in turn translate this sum of "blue side" vector into the sum of 2 sectors of blue side, one " $z_{1} S 1$ " and the other " $z_{2} S 1$ ”. The desired relationship is thus obtained. We will note the consistency with the exponential notation “ $\left(z_{1}+z_{2}\right)\left(R 1 X^{A 1}\right)=\left(z_{1} R 1 X^{A 1}\right)+\left(z_{2} R 1 X^{A 1}\right)$ ".

For the algebraic representation of 2 -tuples of complex numbers, the definition of multiplication by a number gives

$$
\begin{gathered}
\left(z_{4}+z_{3}\right)\left(z_{1}, z_{2}\right)=\left(z_{1},\left(z_{4}+z_{3}\right) z_{2}\right)=\left(z_{1}, z_{4} z_{2}+z_{3} z_{2}\right) \sim\left(z_{1} z_{1}, z_{4} z_{2} z_{1}+z_{3} z_{2} z_{1}\right) \\
z_{4}\left(z_{1}, z_{2}\right)+z_{3}\left(z_{1}, z_{2}\right)=\left(z_{1}, z_{4} z_{2}\right)+\left(z_{1}, z_{3} z_{2}\right)=\left(z_{1} z_{1},\left(z_{4} z_{2}\right) z_{1}+z_{1}\left(z_{3} z_{2}\right)\right)
\end{gathered}
$$

Which verifies the relationship.

\subsubsection{Exponentiation of Sectors by a Number}

Let's show that we have: $S 1^{1}=S 1$. 
For the sector space: Our definition of exponentiation by a number consists in raising to the power of this same number both the complex number representing the "blue side" and the complex number representing the "orange side". The "orange side" being henceforth unitary, its raising in power will not modify it. In our case, it consists in raising the blue side to power 1, which gives an identical blue side sector. The relationship is therefore verified. We will note the consistency with the exponential notation " $\left(R 1 X^{A 1}\right)^{1}=R 1^{1} X^{1 . A 1}=R 1 X^{A 1}$ ”.

For the algebraic representation of 2 -tuples of complex numbers, the definition of exponentiation by a number gives

$$
\left(z_{1}, z_{2}\right)^{1}=\left(z_{1}^{2}, z_{2}^{1}\right)=\left(z_{1}, z_{2}\right)
$$

Which verifies the relationship.

Let's show that we have: $S 1^{0}=1$.

For the sector space: Our definition of exponentiation by a number consists in raising to the power of this same number both the complex number representing the "blue side" and the complex number representing the "orange side". The "orange side" being henceforth unitary, its raising in power will not modify it. In our case, it consists in raising the blue side to power zero, which gives a sector of blue side of unit length, that is to say the sector unit noted 1 . The relation is thus verified. We will note the consistency with the exponential notation " $\left(R 1 X^{A 1}\right)^{0}=R 1^{0} X^{0 . A 1}=1$ ”.

For the algebraic representation of 2-tuples of complex numbers, the definition of exponentiation by a number gives:

$$
\left(z_{1}, z_{2}\right)^{0}=\left(z_{1}^{0}, z_{2}^{0}\right)=(1,1)
$$

Which verifies the relationship.

Let's show that we have: $\left(S 1^{z_{1}}\right)^{z_{2}}=S 1^{z_{1} z_{2}}$

For the sector space: Our definition of exponentiation by a number consists in raising to the power of this same number both the complex number representing the "blue side" and the complex number representing the "orange side". The "orange side" being henceforth unitary, its raising in power will not modify it. In our case, it consists in raising, for the $1^{\text {st }}$ time, the blue side to the power $Z_{1}$, then, for a $2^{\text {nd }}$ time, this new "blue side" to the power $z_{2}$ which in the end consists in directly raising the initial blue side to the power of $z_{1} z_{2}$. This result is actually due to the property of complex numbers (what is the blue side) “ $\left(z^{z_{1}}\right)^{z_{2}}=z^{\left(z_{1} z_{2}\right)}$ ". The relationship is therefore verified. We will note the consistency with the exponential notation

“ $\left(\left(R 1 X^{A 1}\right)^{z_{1}}\right)^{z_{2}}=\left(R 1^{z_{1}} X^{A 1 \cdot z_{1}}\right)^{z_{2}}=R 1^{z_{1} z_{2}} X^{A 1 \cdot z_{1} z_{2}}=\left(R 1 X^{A 1}\right)^{z_{1} z_{2}} »$

Note: It should be noted that if the sides of the sector had been real vectors (and not complex) this relationship would not be verified since the exponentiation of a real number does not always make sense. In other words, the set of numbers associated with our sector space cannot simply be a vector space. We will study further the consequences of this fact and the chain reaction it implies. 
For the algebraic representation of 2-tuples of complex numbers, the definition of exponentiation by a number gives

$$
\left(\left(z_{1}, z_{2}\right)^{z_{3}}\right)^{z_{4}}=\left(z_{1}^{z_{3}}, z_{2}^{z_{3}}\right)^{z_{4}}=\left(z_{1}^{z_{3} z_{4}}, z_{2}^{z_{3} z_{4}}\right)=\left(z_{1}, z_{2}\right)^{z_{3} z_{4}}
$$

Which verifies the relationship.

Let's show that we have: $S 1^{z_{1}+z_{2}}=S 1^{z_{1}} \cdot S 1^{z_{2}}$

For the sector space: As before this comes from a property of complex numbers, namely " $z^{z_{1}+z_{2}}=z^{z_{1}} z^{z_{2}}$ " applied to our "blue side", which then verifies the relationship. We will note the consistency with the exponential notation “ $\left(R 1 X^{A 1}\right)^{z_{1}+z_{2}}=\left(R 1 X^{A 1}\right)^{z_{1}}\left(R 1 X^{A 1}\right)^{z_{2}}$ ”.

IMPORTANT: Again, it should be noted that if the sides of the sector had been real vectors (and not complex) this relationship would not be verified since the exponentiation of a real number does not always make sense.

For the algebraic representation of 2-tuples of complex numbers, the definition of exponentiation by a number gives

$$
\begin{gathered}
\left(z_{1}, z_{2}\right)^{z_{3}+z_{4}}=\left(z_{1}^{z_{3}+z_{4}}, z_{2}^{z_{3}+z_{4}}\right)=\left(z_{1}^{z_{3}} z_{1}^{z_{4}}, z_{2}^{z_{3}} z_{2}^{z_{4}}\right) \\
\left(z_{1}, z_{2}\right)^{z_{3}}\left(z_{1}, z_{2}\right)^{z_{4}}=\left(z_{1}^{z_{3}}, z_{2}^{z_{3}}\right)\left(z_{1}^{z_{4}}, z_{2}^{z_{4}}\right)=\left(z_{1}^{z_{3}} z_{1}^{z_{4}}, z_{2}^{z_{3}} z_{2}^{z_{4}}\right)
\end{gathered}
$$

Which verifies the relationship.

Let's show that we have: $\left(z_{1} S 1\right)^{z_{2}}=z_{1}^{z_{2}} S 1^{z_{2}}$

For the sector space: Multiplying by $z_{1}$ consists in multiplying the vector "blue side" by this number $z_{1}$ (property of vector space). On the other hand, the exponentiation by $z_{2}$ of the blue side again requires, no longer the vector side of complex numbers, but one of their specific properties, namely “ $\left(z_{1} z_{2}\right)^{z}=z_{1}^{z} z_{2}^{z}$ ". Then the definition of $S 1^{z_{2}}$ allows to find the right-hand side of the expression, which then verifies the relation. We will note the consistency with the exponential notation " $\left(z_{1}\left(R 1 X^{A 1}\right)\right)^{z_{2}}=\left(z_{1} R 1 X^{A 1}\right)^{z_{2}}=z_{1}^{z_{2}}\left(R 1 X^{A 1}\right)^{z_{2}}$ ".

Note: Again, a set of numbers which is only a vector space cannot be associated with a sector space.

For the algebraic representation of 2-tuples of complex numbers, the definition of multiplication by a number gives

$$
\begin{aligned}
\left(z_{3}\left(z_{1}, z_{2}\right)\right)^{z_{4}} & =\left(z_{1}, z_{3} z_{2}\right)^{z_{4}}=\left(z_{1}^{z_{4}},\left(z_{3} z_{2}\right)^{z_{4}}\right)=\left(z_{1}^{z_{4}}, z_{3}^{z_{4}} z_{2}^{z_{4}}\right) \\
& =z_{3}^{z_{4}}\left(z_{1}^{z_{4}}, z_{2}^{z_{4}}\right)=z_{3}^{z_{4}}\left(z_{1}, z_{2}\right)^{z_{4}}
\end{aligned}
$$

Which verifies the relationship.

\subsubsection{Distributivity of External Multiplication and Exponentiation over Sector Addition and Sector Multiplication}

Let's show that we have: $z_{1}(S 1 \cdot S 2)=\left(z_{1} S 1\right) \cdot S 2=S 1 \cdot\left(z_{1} S 2\right)$

For the sector space: As for the demonstration of the distributivity of internal composition laws, we will again use the fact that the set of sectors can be bijectively associated with a single complex vector, via the blue side of the sector which firstly identifies univocally any sector and on the other hand which 
represents a vector of the complex vector space which can traverse all the complex space. In this way, by our notation (in which we can take “ $X \sim \mathrm{e}^{i}$ ”):

$$
\begin{aligned}
z_{1}(S 1 \cdot S 2) & \sim z_{1}\left(R 1 X^{A 1} \cdot R 2 X^{A 2}\right)=\left(z_{1} R 1 X^{A 1}\right) \cdot R 2 X^{A 2} \\
& \sim\left(z_{1} S 1\right) \cdot S 2=R 1 X^{A 1} \cdot\left(z_{1} R 2 X^{A 2}\right) \sim S 1 \cdot\left(z_{1} S 2\right)
\end{aligned}
$$

For the algebraic representation of pairs of complex numbers, the definition of sectoral multiplication gives

$$
\begin{gathered}
z_{5}\left(\left(z_{1}, z_{2}\right) \cdot\left(z_{3}, z_{4}\right)\right)=z_{5}\left(z_{1} z_{3}, z_{4} z_{2}\right)=\left(z_{1} z_{3}, z_{5} z_{4} z_{2}\right) \\
\left(z_{5}\left(z_{1}, z_{2}\right)\right) \cdot\left(z_{3}, z_{4}\right)=\left(z_{1}, z_{5} z_{2}\right) \cdot\left(z_{3}, z_{4}\right)=\left(z_{1} z_{3}, z_{4} z_{5} z_{2}\right) \\
\left(z_{1}, z_{2}\right) \cdot\left(z_{5}\left(z_{3}, z_{4}\right)\right)=\left(z_{1}, z_{2}\right) \cdot\left(z_{3}, z_{5} z_{4}\right)=\left(z_{1} z_{3}, z_{5} z_{4} z_{2}\right)
\end{gathered}
$$

Which verifies the relationship.

Let's show that we have: $(S 1 \cdot S 2)^{z_{1}}=S 1^{z_{1}} \cdot S 2^{z_{1}}$.

For the sector space: Let's proceed in the same way as before, with our notation (in which we can take " $X \sim \mathrm{e}^{i}$ ”):

$$
(S 1 \cdot S 2)^{Z_{1}} \sim\left(R 1 X^{A 1} \cdot R 2 X^{A 2}\right)^{z_{1}}=\left(R 1 X^{A 1}\right)^{Z_{1}} \cdot\left(R 2 X^{A 2}\right)^{Z_{1}} \sim S 1^{Z_{1}} \cdot S 2^{z_{1}}
$$

Note: It will be noted that in general the negative exponentiation reverses the order of the elements in the right-hand side $(S 1 \cdot S 2)^{-1}=S 2^{-1} \cdot S 1^{-1}$. Consequently, this relationship implies the commutativity of sectoral multiplication.

For the algebraic representation of pairs of complex numbers, the definition of sectoral multiplication gives

$$
\left(\left(z_{1}, z_{2}\right) \cdot\left(z_{3}, z_{4}\right)\right)^{z_{5}}=\left(z_{1} z_{3}, z_{4} z_{2}\right)^{z_{5}}=\left(z_{1}^{z_{5}} z_{3}^{z_{5}}, z_{4}^{z_{5}} z_{2}^{z_{5}}\right)=\left(z_{1}, z_{2}\right)^{z_{5}}\left(z_{3}, z_{4}\right)^{z_{5}}
$$

Which verifies the relationship.

Let's show that we have: $z_{1}(S 1+S 2)=z_{1} S 1+z_{1} S 2$.

For the sector space: The sectoral addition results in a vector addition of the blue sides. Therefore, this relation comes directly from this same relation verified by the vectors.

For the algebraic representation of 2-tuples of complex numbers, the definition of sectoral addition gives

$$
\begin{aligned}
& z_{5}\left(\left(z_{1}, z_{2}\right)+\left(z_{3}, z_{4}\right)\right)=z_{5}\left(z_{1} z_{3}, z_{2} z_{3}+z_{1} z_{4}\right)=\left(z_{1} z_{3}, z_{5} z_{2} z_{3}+z_{5} z_{1} z_{4}\right) \\
& z_{5}\left(z_{1}, z_{2}\right)+z_{5}\left(z_{3}, z_{4}\right)=\left(z_{1}, z_{5} z_{2}\right)+\left(z_{3}, z_{5} z_{4}\right)=\left(z_{1} z_{3}, z_{5} z_{2} z_{3}+z_{1} z_{5} z_{4}\right)
\end{aligned}
$$

Which verifies the relationship.

To be systematic in the combinations of the different operations, there are 2 more cases to study $(S 1)^{z_{1}^{z_{2}}}$ and $(S 1+S 2)^{z_{1}}$.

For the $1^{\text {st }}$ expression, there is a priori no relation associating it with an equivalent expression.

For the $2^{\text {nd }}$ expression, we can notice that by the previous rules, $(S 1+S 2)^{Z_{1}}=$ $(S 1+S 2) \cdot(S 1+S 2)^{z_{1}-1}=S 1 \cdot(S 1+S 2)^{z_{1}-1}+S 2 \cdot(S 1+S 2)^{z_{1}-1}$ and $(S 1+S 2)^{z_{1}}=$ $S 1^{z_{1}} \cdot(1+S 2 / S 1)^{z_{1}}$. We can therefore restrict this formulation to $(1+S 1)^{z_{1}}$ with 
$\left.\left\|z_{1}\right\| \in\right] 0 ; 1\left[\right.$. As for the $1^{\text {st }}$ expression, there does not exist a priori a relation associating it with an equivalent expression (except to consider the infinite limited developments). We will also see that the terms " $1+S 1$ " could play a structuring role in the structures that we are going to define. Consequently, it is not impossible to think that there is no rule of distributivity of the exponentiation over the addition, thus characterizing a form of irreducibility of this expression. Therefore, in our proposal for nonlinear structures, we will assume that there are no rules associated with these 2 expressions.

\subsubsection{Consequences of Internal and External Laws on the Set of Numbers Associated with the Sector Space}

So far, we have focused our study on the sectoral space itself. We will now study the set of numbers associated with our sector space (in this case the set of complex numbers). Implicitly, the 4 laws of composition, internal and external, imply that the associated set has 3 laws of internal composition (addition, multiplication and exponentiation). We see this when we write for example the following relationships:

$$
\left(z_{1}+z_{2}\right) S 1=z_{1} S 1+z_{2} S 1 ;\left(z_{1} z_{2}\right) S 1=z_{1}\left(z_{2} S 1\right) ;\left(z_{1} S 1\right)^{z_{2}}=z_{1}^{z_{2}} S 1^{z_{2}}
$$

Then, for reasons of compatibility between the laws of space and the laws of the associated set, the properties of our space explicitly lead to similar properties in the set which is associated with it. Thus, the internal addition on the associated set must form a group because the sectoral addition forms a group and we have the following relationships:

$\left(z_{1}+z_{2}\right) S 1=z_{1} S 1+z_{2} S 1 ; 0 \cdot S 1=0$; The existence of an opposite " $-S 1$ ".

In the same way, the internal multiplication on the associated set must form a group because the sectoral multiplication forms a group and we have the following relationships:

$\left(z_{1} z_{2}\right) S 1=z_{1}\left(z_{2} S 1\right) ; 1 \cdot S 1=S 1$; The existence of an inverse " $S 1^{-1}$ ".

In addition, we used the vector space properties on a field of complex numbers to demonstrate certain properties of the sector addition (which corresponded to a vector addition on the "blue side" vector). Consequently, the internal composition law "+" (addition in the associated set) and the internal composition law ". "(Multiplication in the associated set) must form a field. We can also convince ourselves with these relationships:

$$
z_{3}(S 1+S 2)=z_{3} S 1+z_{3} S 2 \text { with } S 1=z_{1} S 0 \text { et } S 2=z_{2} S 0
$$

For the $3^{\text {rd }}$ law of internal composition “ $\wedge$ ” (exponentiation), we had to explicitly use the 2 following properties:

$$
\left(z_{1} z_{2}\right)^{z}=z_{1}^{z} z_{2}^{z} ; z^{z_{1}+z_{2}}=z^{z_{1}} z^{z_{2}}
$$

To be complete, we must also add the following relationships: $z_{1}^{0}=1$.

Because we need it when we associate the following 2 relationships:

$$
\left(z_{1} S 1\right)^{z_{2}}=z_{1}^{z_{2}} S 1^{z_{2}} ; S 1^{0}=1 ; z_{1}^{1}=z_{1}
$$


Because we need it when we associate the following 2 relationships:

$$
\left(z_{1} S 1\right)^{z_{2}}=z_{1}^{z_{2}} S 1^{z_{2}} ; S 1^{1}=S 1 ;\left(z_{1}^{z_{2}}\right)^{z_{3}}=z_{1}^{z_{2} z_{3}}
$$

Because we need it when we associate the following 2 relationships:

$$
\left(z_{1} S 1\right)^{z_{2}}=z_{1}^{z_{2}} S 1^{z_{2}} ; S 1^{z_{2} z_{3}}=\left(S 1^{z_{2}}\right)^{z_{3}}
$$

As in the previous section, to be systematic in the combinations of the different operations, we would need the last 2 relations which are associated with $(S 1)^{z_{1}^{z_{2}}}$ and $(S 1+S 2)^{z_{1}}$

$$
\left(z_{1}\right)^{z_{2}^{z_{3}}} \text { et }\left(z_{1}+z_{2}\right)^{z_{3}}
$$

But for the same reasons as before, in our proposal for nonlinear structures, we will assume that there are no rules associated with these 2 expressions (implying that they could be associated with a form of irreducibility that we treat a little further).

\section{Nonlinear Algebraic Structures}

\subsection{On the Way to a Succession of Algebraic Structures Associated with the Sectoral Space}

Let us make a $1^{\text {st }}$ synthesis of the properties of our sectoral space and its associated set of numbers in order to extract from it abstract structures which will go beyond the "linear" structures of vector space and of field, reason for which we will name them non-linear. At this stage, 2 structures (beyond vector space and field which will therefore be named non-linear space and non-linear field) emerge from the above, but this $1^{\text {st }}$ synthesis will lead us to consider a new structure of group.

\subsubsection{Sector Space or Non-Linear Space (ENL)}

The sectoral space forms a set A provided with two internal composition laws, the sectoral addition denoted "+" and the sectoral multiplication denoted ".":

The internal composition laws "+" and "." form a commutative field on A.

The sector space is associated with a set B provided with three internal composition laws “+”, “.” and “ $\wedge$ ” (exponentiation):

The internal composition laws "+” and "." form a commutative field on B.

The internal composition law “ $\wedge$ ” (exponentiation) verifies:

$$
z_{1}^{0}=1 ; z_{1}^{1}=z_{1} ; z^{z_{1}+z_{2}}=z^{z_{1}} z^{z_{2}} ; z_{1}^{z_{2} z_{3}}=\left(z_{1}^{z_{2}}\right)^{z_{3}} ;\left(z_{1} z_{2}\right)^{z}=z_{1}^{z} z_{2}^{z}
$$

We have provided this space with two external composition laws “.” and “^” (exponentiation) which verify:

$$
\begin{aligned}
& 0 \cdot S 1=0 ; 1 \cdot S 1=S 1 ;\left(z_{1} z_{2}\right) S 1=z_{1}\left(z_{2} S 1\right) ;\left(z_{1}+z_{2}\right) S 1=z_{1} S 1+z_{2} S 1 \\
& S 1^{1}=S 1 ; S 1^{0}=1 ; S 1^{z_{1} z_{2}}=\left(S 1^{z_{1}}\right)^{z_{2}} ; S 1^{z_{1}+z_{2}}=S 1^{z_{1}} \cdot S 1^{z_{2}} ;\left(z_{1} S 1\right)^{z_{2}}=z_{1}^{z_{2}} S 1^{z_{2}} \\
& z_{1}(S 1+S 2)=z_{1} S 1+z_{1} S 2 ; z_{1}(S 1 \cdot S 2)=\left(z_{1} S 1\right) \cdot S 2=S 1 \cdot\left(z_{1} S 2\right) \\
& (S 1 \cdot S 2)^{z_{1}}=S 1^{z_{1}} \cdot S 2^{z_{1}}
\end{aligned}
$$




\subsubsection{Non-Linear Field (CNL)}

From the above, we can define a new structure (the non-linear equivalent of the notion of field):

Consider a set B provided with three internal composition laws "+", “.” and “^" (exponentiation):

The internal composition laws "+" and “." form a commutative field on B.

The internal composition law " $\wedge$ ” (exponentiation) verifies:

$$
z_{1}^{0}=1 ; \quad z_{1}^{1}=z_{1} ; z^{z_{1}+z_{2}}=z^{z_{1}} z^{z_{2}} ; z_{1}^{z_{2} z_{3}}=\left(z_{1}^{z_{2}}\right)^{z_{3}} ;\left(z_{1} z_{2}\right)^{z}=z_{1}^{z} z_{2}^{z}
$$

\subsubsection{Non-Linear Group (LNG)}

Different considerations lead us to consider the existence of a group type structure. The most fundamental reason is related to what we will see a little later, the sectoral space is to projective space what is vector space to affine space. The link between affine space and vector space is made through affine applications whose generic expression is " $x^{\prime}=a x+b$ ", but the notion of group ensures the existence of such expressions. The connection between projective space and sectoral space will be carried out through projective applications or homographies (we will see that the sectors are a representation of it) whose generic expression is “ $x^{\prime}=(a x+b) /(c x+d)$ ". In order to ensure the existence of these expressions, we can expect the need of a new notion of group. In addition, this expression is associated with the following form " $x^{\prime}(c x+d)=(a x+b)$ " or " $A x x^{\prime}+B x^{\prime}+C=0$ ”. We can therefore even foresee that this new group will have to make sense of the $2^{\text {nd }}$ degree equations. A $2^{\text {nd }}$ is that it is quite astonishing that the relation $(S 1+S 2)^{z_{1}}$ has no explicit rewriting rules. However, from the rules already established, we can modify this expression, which has the consequence of reducing this problem to $(1+S 1)^{z_{1}}$ with $\left.\left\|z_{1}\right\| \in\right] 0 ; 1[$ in which only one variable sector intervenes. Consequently, one way of explaining this absence of rewriting could be that the element of the form " $1+S 1$ " could be a specific structuring element (by its irreducibility). The definition of such new specific elements necessarily implies a new group type structure.

A third consideration comes from the fact that, even if this may not be reflected in this study, we put these structures in place by taking the "Vector Space-Field-Group" scheme and making them evolve systematically ( $\mathrm{N}$ linear composition laws $->(\mathrm{N}+1)$ non-linear composition laws, minimum dimension $1 \mathrm{D}$ in linear $->2 \mathrm{D}$ minimum dimension in non-linear, segment/vector in linear -> parallelogram/sector in non-linear, 1 center and 2 diametrically opposed elements on a linear circle $->2$ focuses and 4 elements on a conic, ...). So, from this point of view, we can define the vector space as follows. Consider a set A with an internal composition law "+". The internal composition law on A forms a group. Consider a set B provided with two internal composition laws " + " and “.”. The two internal composition laws on B form a field. Consider an external composition law “.”. A on B forms a vector space (linear). Which would become for our sector space. Consider a set A provided with two internal composition laws "+" 
and ".". The two internal composition laws on A form a non-linear group. Consider a set B provided with three internal composition laws "+" and "." and " $\wedge$ " (exponentiation). The three internal composition laws on B form a non-linear field. Consider two external composition laws “.” and " $\wedge$ " (exponentiation). A on $\mathrm{B}$ forms a sectoral space (non-linear). And it emerges from the previous study that we have effectively defined a sectoral space and a nonlinear field. By this procedure of extension of the linear, it would then remain to formalize a nonlinear group. Finally, a fourth consideration comes from the fact that as we will see a little later, these structures have to do with domains that do not fall within the linear domain. Thus, we will see that the sectoral space is linked to the projective spaces, that in the sector there are elements of the modular group [3]. Characteristics of dynamical systems and arithmetic will also appear in this sectoral space structure. All these areas show the need to go beyond the linear notions originating from the group (punctual elements, binary relationships, ... are notions incapable of accessing non-linear behaviors such as baker's map, self-similarity, ...). Obtaining such fundamental elements requires the development of a group type structure which, unlike the other kind of structures, allows explicitly revealing elements specific of a considered domain (linear or not). This is how the notion of group is the basis of all linear tools. In our opinion, a notion of group specific to the non-linear which extends the linear group is necessary.

Non-linear group proposal

As indicated previously, a possible way to propose a notion of non-linear group would be to start from the notion of group in order to extend it. The latter has the characteristic of being associated:

- "Geometrically" to the concept of circle/line with 1 center (the neutral element) and 2 diametrically opposite elements (an element and its opposite) on this circle.

- "Algebraically" to the ability to give meaning to the writing " $z+a=0$ " then to " $b z+a=0$ ".

Let's look for an associated structure:

"Geometrically" to the concept of conic (ellipse, parabola and hyperbola) with 2 focuses and 4 elements arranged on this conic.

"Algebraically" to the ability to give meaning to the writing " $z^{2}+a=0$ ", “ $z^{2}+b z+a=0$ ” then to “ $c z^{2}+b z+a=0$ ”.

These $2^{\text {nd }}$ degree equations can be written more generally, on a commutative field, in the form $\left(Z-z_{1}\right)\left(Z-z_{2}\right)=Z^{2}-\left(z_{1}+z_{2}\right) Z+\left(z_{1} z_{2}\right)=0$ with $z_{1}$ and $Z_{2}$ the elements which must be ensured to give meaning to this writing. We must therefore ensure that:

$$
\forall(a, b) \exists\left(z_{1}, z_{2}\right) \text { tel que }\left\{\begin{array}{l}
-b=z_{1}+z_{2} \\
a=z_{1} z_{2}
\end{array}\right.
$$

We have previously seen that our space formed a group for addition, so we will simplify the writing by replacing " $-b$ " with " $b$ ". The idea is now to restrict 
this expression to only the structuring elements, that is to say not to consider all the 2 -tuples $(a, b)$. Structurally, we have only 2 known elements, the neutral element of the addition "0" and that of the multiplication " 1 ". We then have 4 possible expressions (we noted the element $a$ or $b$ by $z$ ):

$$
\begin{aligned}
& \forall z \exists\left(z_{1}, z_{2}\right) \text { tel que }\left\{\begin{array}{l}
1=z_{1}+z_{2} \\
z=z_{1} z_{2}
\end{array}\right. \\
& \forall z \exists\left(z_{1}, z_{2}\right) \text { tel que }\left\{\begin{array}{l}
0=z_{1}+z_{2} \\
z=z_{1} z_{2}
\end{array}\right. \\
& \forall z \exists\left(z_{1}, z_{2}\right) \text { tel que }\left\{\begin{array}{l}
z=z_{1}+z_{2} \\
1=z_{1} z_{2}
\end{array}\right. \\
& \forall z \exists\left(z_{1}, z_{2}\right) \text { tel que }\left\{\begin{array}{l}
z=z_{1}+z_{2} \\
0=z_{1} z_{2}
\end{array}\right.
\end{aligned}
$$

The first 2 formulations make it possible to give meaning to all of the equations of degree 2. The $1^{\text {st }}$ formulation is centered on the focus " 1 " while the $2^{\text {nd }}$ formulation is centered on the focus "0". And we will notice that the characteristic elements that they highlight (i.e. complementary and square root) are linked.

$1^{\text {st }}$ non-linear group formulation.

Let us demonstrate that the $1^{\text {st }}$ formulation makes it possible to ensure a meaning for all the equations of the $2^{\text {nd }}$ degree:

1) This relation directly implies that the following equations always have a meaning and more precisely that they have 2 solutions $Z=z_{1}$ et $Z=z_{2}$ :

$$
\forall a \exists\left(z_{1}, z_{2}\right) \text { such as }\left\{\begin{array}{l}
1=z_{1}+z_{2} \\
a=z_{1} z_{2}
\end{array} \Leftrightarrow Z^{2}-Z+a=0\right.
$$

To get the solutions of

$$
Z^{2}+b Z+a=0 \text { with } b \neq 0
$$

It is enough to know the solutions of

$$
Z^{2}-Z+\frac{a}{b^{2}}=0 \text { with } b \neq 0
$$

What is ensured by the expression of the non-linear group:

$$
\forall A \exists\left(z_{1}, z_{2}\right) \text { such as }\left\{\begin{array}{l}
1=z_{1}+z_{2} \\
A=\frac{a}{b^{2}}=z_{1} z_{2}
\end{array}\right.
$$

And since the non-linear group is a group for addition and multiplication we can write any element $A$ in the form $A=-\frac{a}{b^{2}}$.

We can then write (by multiplying 1 time by “ $-b$ " the $1^{\text {st }}$ relation and 2 times by " $-b$ " the $2^{\text {nd }}$ ):

$$
\forall(a, b) \exists\left(z_{1}, z_{2}\right) \text { such as }\left\{\begin{array}{l}
-b=\left(-b z_{1}\right)+\left(-b z_{2}\right) \\
a=\left(-b z_{1}\right)\left(-b z_{2}\right)
\end{array}\right.
$$


That to say:

$$
Z^{2}+b Z+a=\left(Z+b z_{1}\right)\left(Z+b z_{2}\right)=0 \text { with } b \neq 0
$$

2) For the case:

$$
c Z^{2}+b Z+a=0 \text { with } c \neq 0
$$

These solutions are simply obtained by dividing by $c$.

$$
Z^{2}+\frac{b}{c} Z+\frac{a}{c}=0 \text { with } c \neq 0
$$

With the above, we know that this relationship has a meaning (for $b \neq 0$ ).

3) For the case:

$$
Z^{2}+a=0
$$

We know that

$$
\forall c \quad \exists z_{1} \text { and } z_{2} \text { such as } Z^{\prime 2}-Z^{\prime}+c=0
$$

If we set $Z^{\prime}=Z+d$, we can then write

$$
\forall c \exists\left(z_{1}-d\right) \text { and }\left(z_{2}-d\right) \text { such as }(Z+d)^{2}-(Z+d)+c=0
$$

That we can write

$$
Z^{2}+Z(2 d-1)+d(d-1)+c=0
$$

Then just take $d=\frac{1}{2}$ to cancel the term in $Z$ and choose $c=a-d$ $(d-1)=a+1 / 4$.

The value of $c$ makes it possible to define the equation in $Z^{\prime}$ and its solutions $z_{1}$ and $z_{2}$. The solutions of $Z^{2}+a=0$ are then $z_{1}-d$ et $z_{2}-d$.

$2^{\text {nd }}$ non-linear group formulation

Let us demonstrate that the $2^{\text {nd }}$ formulation also makes it possible to ensure a meaning for all the equations of the $2^{\text {nd }}$ degree.

1) This relation directly implies that the following equations always have a meaning and more precisely that they have 2 solutions $Z=z_{1}$ and $Z=z_{2}$ :

$$
\forall a \exists\left(z_{1}, z_{2}\right) \text { such as }\left\{\begin{array}{l}
0=z_{1}+z_{2} \\
a=z_{1} z_{2}
\end{array} \Leftrightarrow Z^{2}+a=0\right.
$$

We can apply a similar approach to the previous one.

We know that

$$
\forall c \quad \exists z_{1} \text { and } z_{2} \text { such as } Z^{\prime 2}+c=0
$$

If we set $Z^{\prime}=Z+d$, we can then write

$$
\forall c \exists\left(z_{1}-d\right) \text { and }\left(z_{2}-d\right) \text { such as }(Z+d)^{2}+c=0
$$

That we can write:

$$
Z^{2}+2 d Z+d^{2}+c=0
$$

It is then enough to take $d=-\frac{1}{2}$ to obtain the equation of the $1^{\text {st }}$ formula- 
tion of the non-linear group with the term in $Z$ equal to " -1 " and to choose $c=a-d^{2}=a-1 / 4$. The value of $c$ makes it possible to define the equation in $Z^{\prime}$ and its solutions $z_{1}$ and $z_{2}$. The solutions of $Z^{2}-Z+a=0$ are then $z_{1}-d$ and $z_{2}-d$. The other cases are obtained in the same way as for those studied in the $1^{\text {st }}$ formulation of the non-linear group. Thus, these 2 expressions are sufficiently structuring to provide a meaning to all the equations of the $2^{\text {nd }}$ degree. This is not the case for the other 2 formulations.

The $3^{\text {rd }}$ expression provides a meaning for the equation " $Z^{2}-b Z+1=0$ " with $b$ different from 0 but it does not guarantee from the addition and multiplication a meaning for all the equations of the $2^{\text {nd }}$ degree (without the addition of other characteristics). Indeed, the multiplication by $b$ as we did for the $1^{\text {st }}$ case occurs in " $1=z_{1} z_{2}$ " which requires beforehand to pose the existence of $b^{1 / 2}$ to be able to write " $1=\left(b^{1 / 2} z_{1}\right)\left(b^{1 / 2} z_{2}\right)$ ". It is the same from the addition when trying to use “ $Z$ ' $=Z+d$ ". The $4^{\text {th }}$ expression using the absorbing element of multiplication does not provide a meaning for most of the desired equations. We deduce from these last remarks that the first 2 expressions appear as strongly structuring. Note that these expressions can be expressed in a more compact way:

For the $1^{\text {st }}$ formulation: $\forall z \exists z_{1}$ such as $z=z_{1}\left(1-z_{1}\right)$

For the $2^{\text {nd }}$ formulation: $\forall z \exists z_{1}$ such as $z=z_{1}\left(0-z_{1}\right)=-z_{1}^{2}$

We note in passing the similarity of the two expressions " $z=z_{1}\left(e-z_{1}\right)$ " with the neutral element of one or the other of the composition laws. According to the $1^{\text {st }}$ formulation, the existence of such a group corroborates what we imagined to explain the absence of the relation for $(S 1+S 2)^{z_{1}}$ which is associated with the writing $(1+S 1)^{z_{1}}$ ". And the $2^{\text {nd }}$ formulation could also corroborate what we imagined to explain the absence of the relation for $\left(z_{1}\right)^{z_{2}^{z_{3}}}$. In this $2^{\text {nd }}$ formulation, it is the element of the type $\left(z_{1}\right)^{z_{2}^{-1}}$ which would be associated with the writing “ $\left(z_{1}\right)^{z_{2}^{z_{3}}}$ ”.

Uniqueness of the decomposition into an element and its complementary

Let us show the uniqueness of the decomposition into " $z(1-z)$ ".

We have

$$
\forall z \exists z_{1} \text { such as } z=z_{1}\left(1-z_{1}\right)
$$

Assume that there is another element that verifies the same relationship

$$
\forall z \exists z_{3} \neq z_{1} \text { such as } z=z_{3}\left(1-z_{3}\right)
$$

We then deduce that we have:

$$
z_{3}\left(1-z_{3}\right)=z_{1}\left(1-z_{1}\right) ;\left(z_{3}-z_{3}^{2}\right)=\left(z_{1}-z_{1}^{2}\right) ;\left(z_{3}-z_{1}\right)=\left(z_{3}^{2}-z_{1}^{2}\right)
$$

And finally

$$
\left(z_{3}-z_{1}\right)=\left(z_{3}-z_{1}\right)\left(z_{3}+z_{1}\right)
$$

We then have 2 cases:

$$
\text { if } \begin{aligned}
z_{3} \neq z_{1} \text { then }\left(z_{3}+z_{1}\right) & =1 \text { that is } z_{3}=1-z_{1} \\
\text { if not } z_{3} & =z_{1}
\end{aligned}
$$


In the 2 cases we thus find the 2 elements $z_{1}$ and $1-z_{1}$ which proves the uniqueness of our expression.

Let's also show the uniqueness of the decomposition into " $-z^{2}$ " for the $2^{\text {nd }}$ formulation.

We have:

$$
\forall z \exists z_{1} \text { such as } z=-z_{1}^{2}
$$

Assume that there is another element that verifies the same relationship:

$$
\forall z \exists z_{3} \neq z_{1} \text { such as } z=-z_{3}^{2}
$$

We then deduce that we have:

$$
z_{3}^{2}=z_{1}^{2} ;\left(z_{3}^{2}-z_{1}^{2}\right)=0
$$

And finally

$$
\left(z_{3}-z_{1}\right)\left(z_{3}+z_{1}\right)=0
$$

We then have 2 cases:

$$
z_{3}=z_{1} \text { or } z_{3}=-z_{1}
$$

In the 2 cases we thus find the 2 elements $z_{1}$ and $-z_{1}$ which proves the uniqueness of our expression.

\section{Remarkable elements}

We expected the non-linear group to make sense to the $2^{\text {nd }}$ degree equations. This is what led us to the previous definitions. But we also hoped that 2 focuses and 4 elements on the conic would appear. We can see from this point of view that the $1^{\text {st }}$ formulation seems closer to what we expected. The 2 focuses actually appear, both as the neutral elements of the 2 internal composition laws (in this case 0 for addition and 1 for multiplication) but also with their explicit link in the expression " $z_{1}\left(1-z_{1}\right)$ " for which these 2 focuses are 2 "poles" of this expression. This is one of the reasons why we would favor this formulation. We also hoped to obtain 4 elements which would characterize the conic. If we look at the notion of linear group associated with the vector element which is similar to a segment, we notice that this fundamental segment can be seen in one direction or the other depending on whether we take the origin on either side of the segment. This freedom of choice has the consequence of providing the 2 possible types of fundamental elements of the group, " $z$ " and " $-z$ ". Let us use the same principle on our parallelogram "sector" and look at all the sectors which it is possible to determine according to whether one takes as origin one of the four ends and according to whether one takes one side or the other of this origin to define the reference side:

We thus obtain the elements of type " $Z$ ”, “ $-Z$ ” and " $Z$ "-1" and their combination (Figure 21). But this sector can also define sectors whose blue side or orange side are on the diagonals.

This gives us the $4^{\text {th }}$ new element " $1-Z$ ” (Figure 22 ), the other variants producing sectors which are expressed by combination of these 4 elements. By this 


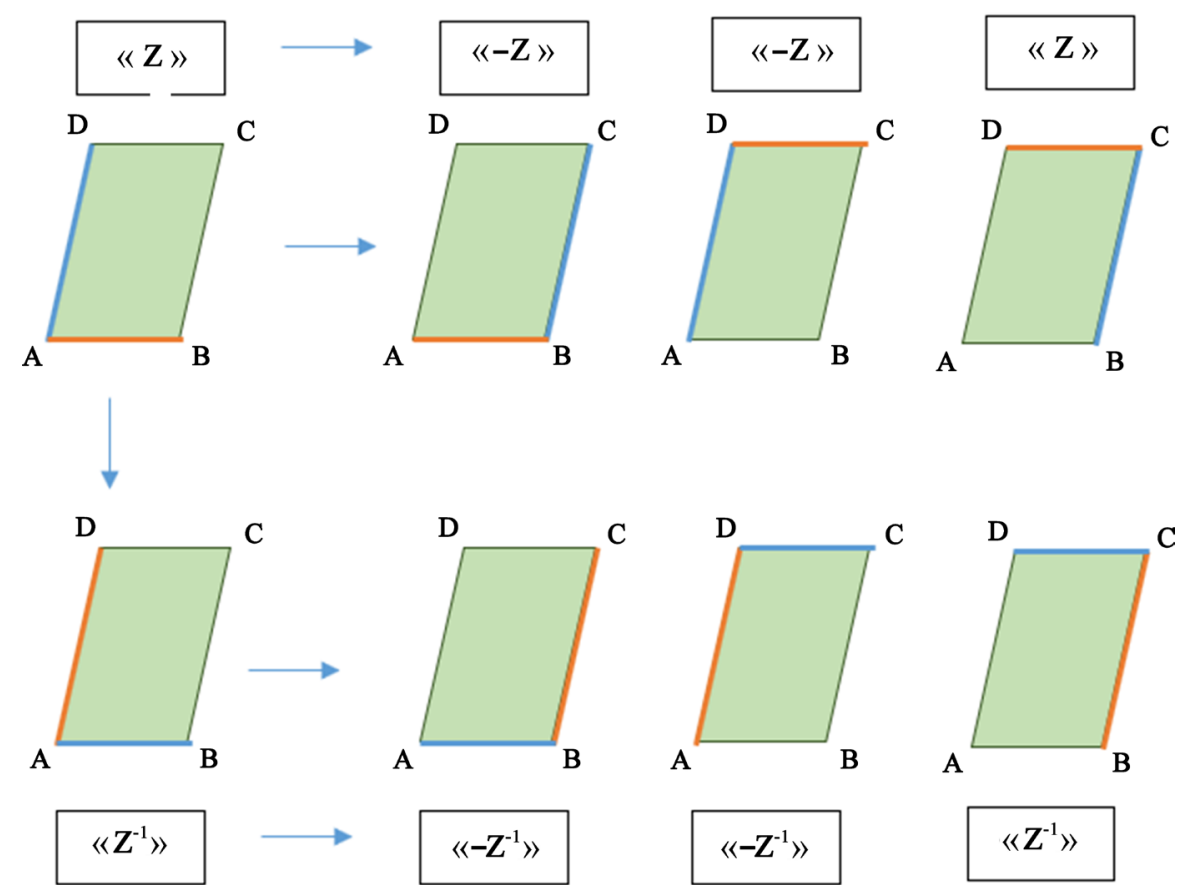

Figure 21. Combinations for connecting the blue and orange sides at four points of a parallelogram.

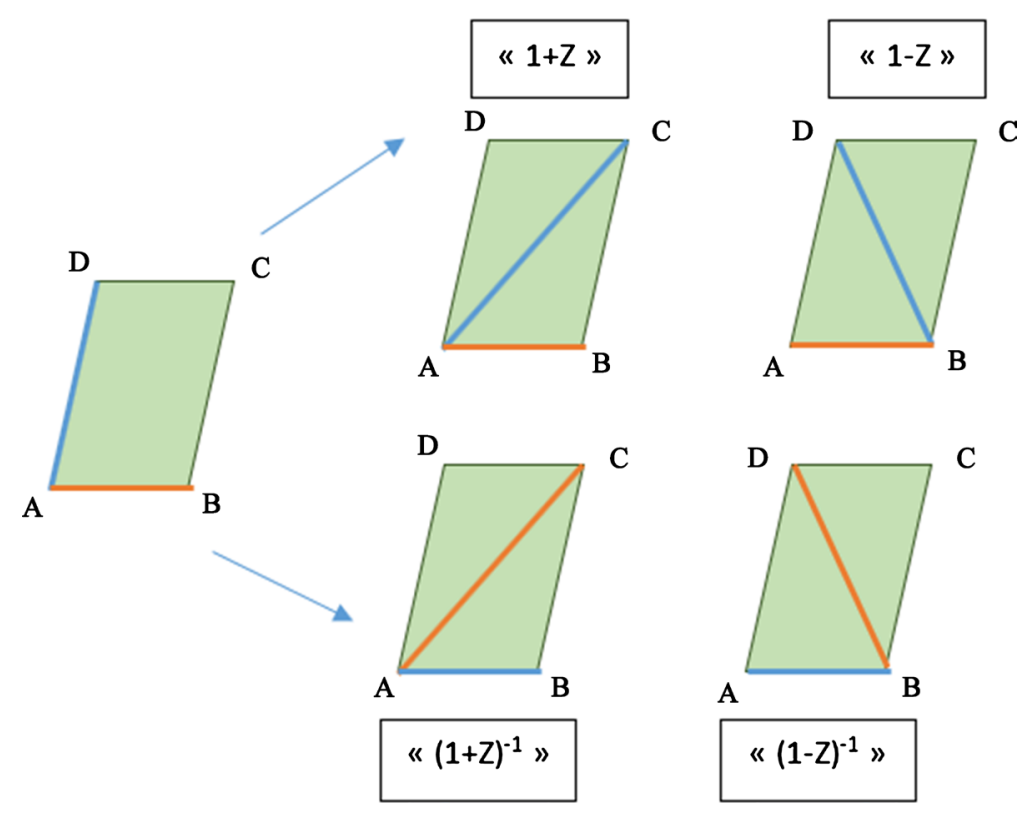

Figure 22. Combinations for connecting the blue and orange sides at three points of a parallelogram.

procedure we find again the 4 expected elements which are those which we defined in our non-linear group: the element ' $Z$ ', its opposite " $-Z$ ' by the addition group, its inverse " $Z^{-1}$ " by the group for multiplication, its complementary " $1-$ $Z$ ' by the non-linear group. With this principle, we could even find the specific elements (neutral and/or focuses). For the sector (A, B, C, D) which can be noted in bi-vectors $((A, B),(A, D))$, these would be the following cases $((A, B)$, 
$(A, B))=1$ and $((A, B),(A, A))=0$. We can also notice that for the blue side sectors of unit length, the inverse corresponds to a form of conjugate (in the sense that in association with the set of complexes, this inverse is the conjugate complex). External multiplication then makes it possible to obtain the set of conjugates (in this case by multiplying by real numbers) from sectors whose ends on the blue side lie on the unit circle. Using this geometric representation, we can see how the notions of complement and square root are linked. Indeed, " $1-z$ " is an element that passes through the diagonal of the parallelogram, its existence is then intimately linked to the hypotenuse of the triangles that make up this sector and therefore to the existence of square roots. We can see it better if we take rectangular sectors. And the successive application of this term provides an infinity of them.

\subsection{Synthesis of Definitions of Nonlinear Structures}

\subsubsection{Non-Linear Group (LNG)}

The non-linear group forms a set A provided with two internal composition laws, the addition denoted " + " and the multiplication "." (the point may not be shown):

The internal composition laws "+" and "." form a commutative field on A.

Every element has a complementary element:

$$
\forall z \exists\left(z_{1}, z_{2}\right) \text { such as }\left\{\begin{array}{l}
1=z_{1}+z_{2} \\
z=z_{1} z_{2}
\end{array}\right.
$$

and more compactly:

$$
\forall z \exists z_{1} \text { such as } z=z_{1}\left(1-z_{1}\right)
$$

Or in a dual way:

$$
\forall z \exists\left(z_{1}, z_{2}\right) \text { such as }\left\{\begin{array}{l}
0=z_{1}+z_{2} \\
z=z_{1} z_{2}
\end{array}\right.
$$

and more compactly:

$$
\forall z \exists z_{1} \text { such as } z=z_{1}\left(0-z_{1}\right)=-z_{1}^{2}
$$

Despite these 2 possible facets of LNG, our preference goes to the $1^{\text {st }}$ expression because it highlights this new element " $1-z$ " and especially this mixture, this composition " $z(1-z)$ ” which appears to us as the crucial element of these new structures. In our opinion, this is the main entrance to the non-linear domain (as we explain below with the notions of extended object and ternary structuring).

\subsubsection{Non-Linear Field (CNL)}

The non-linear field forms a set B provided with three internal composition laws “+”, “.” and “ $\wedge$ ” (exponentiation noted as $\left.a^{b}\right)$ :

The internal composition laws " + " and "." form a non-linear group on B.

The internal composition law “ $\wedge$ ” (exponentiation) verifies 


$$
z_{1}^{0}=1 ; \quad z_{1}^{1}=z_{1} ; z^{z_{1}+z_{2}}=z^{z_{1}} z^{z_{2}} ; z_{1}^{z_{2} z_{3}}=\left(z_{1}^{z_{2}}\right)^{z_{3}} ;\left(z_{1} z_{2}\right)^{z}=z_{1}^{z} z_{2}^{z}
$$

\subsubsection{Sectoral Space (ENL)}

The sectoral space forms a set A provided with two internal composition laws, the sectoral addition denoted "+" and the sectoral multiplication denoted ".":

The internal composition laws "+" and "." form a non-linear group on A.

The sectoral space is associated with a set $B$ provided with three internal composition laws “+”, “.” and “^” (exponentiation):

The internal composition laws “+”, “.” and “^” (exponentiation) form a non-linear field on $\mathrm{B}$.

The sectoral space is provided with two external composition laws "." and “^” (exponentiation) which verify:

$$
\begin{array}{rl}
0 & S 1=0 ; 1 \cdot S 1=S 1 ;\left(z_{1} z_{2}\right) S 1=z_{1}\left(z_{2} S 1\right) ;\left(z_{1}+z_{2}\right) S 1=z_{1} S 1+z_{2} S 1 \\
S 1^{1}= & S 1 ; S 1^{0}=1 ; S 1^{z_{1} z_{2}}=\left(S 1^{z_{1}}\right)^{z_{2}} ; S 1^{z_{1}+z_{2}}=S 1^{z_{1}} \cdot S 1^{z_{2}} ;\left(z_{1} S 1\right)^{z_{2}}=z_{1}^{z_{2}} S 1^{z_{2}} \\
& z_{1}(S 1+S 2)=z_{1} S 1+z_{1} S 2 ; z_{1}(S 1 \cdot S 2)=\left(z_{1} S 1\right) \cdot S 2=S 1 \cdot\left(z_{1} S 2\right) ; \\
& (S 1 \cdot S 2)^{z_{1}}=S 1^{z_{1}} \cdot S 2^{z_{1}}
\end{array}
$$

\subsubsection{Base and Dimension}

One of the strengths of the concept of vector space is its ability to extend to spaces of dimension greater than 1 (its minimum dimension). We can therefore wonder about this characteristic for our sector space. A first element of response is that we can also develop our sectors in larger spaces. For example, in $3 \mathrm{D}$, it suffices a priori to fix the origin and an axis which will correspond to the orange reference side. Then the blue side can move in $3 \mathrm{D}$ space (Figure 23), to form sectors (which always remain $2 \mathrm{D}$ parallelograms).

A $2^{\text {nd }}$ element of response is to look at the definition of a linear base for which

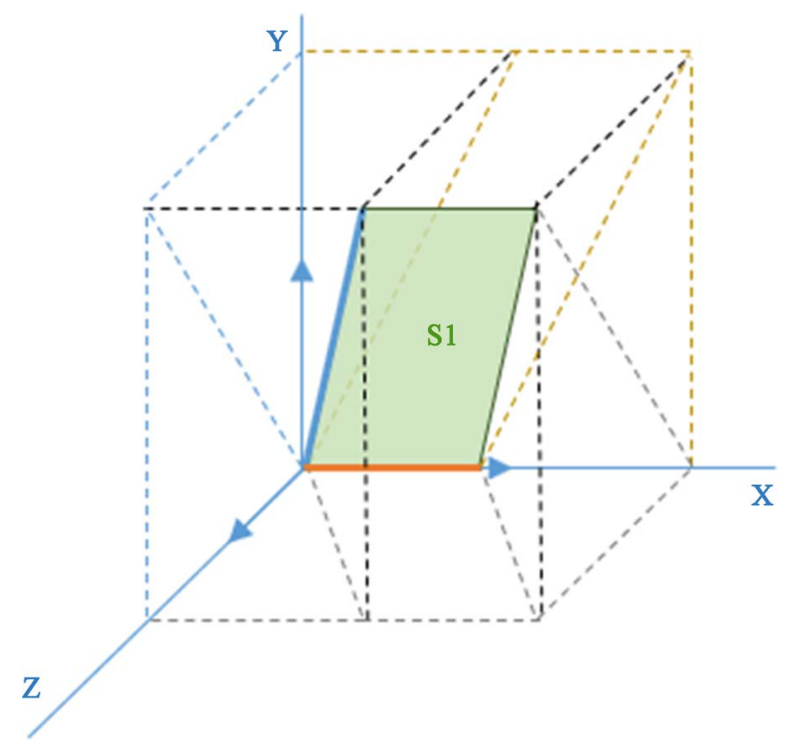

Figure 23. Sector in 3D space. 
it is a question of defining linear independence in this way

$$
a X+b Y=0 \Leftrightarrow a=b=0
$$

In our sectoral space, we can extend this definition to the arithmetic notion of primality [4] in the form:

$$
X^{a} \cdot Y^{b}=1 \Leftrightarrow a=b=0
$$

So, in this sectoral space, we have a priori 2 possible notations, in the form of a linear combination or else in the form of a prime factor decomposition. We also find this specificity in the space of complex numbers, with " $z=1 \cdot x$ $+i \cdot y=r \mathrm{e}^{i \alpha}$ ". This new definition of the base thus generates a link between our non-linear structures and arithmetic (notion of prime factor and irreducibility). These 2 bases can be made up of the same sectors as seen immediately after (thus forming only one base). The formulation with multiplicative coefficients is to be associated with the sector addition. The formulation with the exponents is to be associated with the sectoral multiplication. The multiplicative coefficient "lengthens" the base sector in the direction of the "blue" side. The exponent "rotates" the base sector around the origin and in the plane of the base sector (except for the flat sector which carries the only multiplicative coefficient of this formulation and of which the exponent has no effect when it is normalized because it represents the unit).

Base according to the linear decomposition (additive decomposition):

Let's take a closer look at how we can define a basis in which a sector would have a unique determination. Like a 2-dimensional orthonormal vector base, for which 2 perpendicular lines passing through the origin and carried by normalized vectors make it possible to provide a unique vector decomposition, we can define (for a sectoral base in 3D) 2 perpendicular planes passing through the orange side (such as the $X Z$ and $X Y$ planes in Figure 24) carried by square sectors with normalized sides, $S b_{X Y}$ and $S b_{X Z}$ as in Figure 24. These 2 sectors of base allow to obtain any sector whose blue side is anywhere in the $Y Z$ plane. It is therefore still necessary to add a last sector which makes it possible to move (if necessary) this blue side outside the $Y Z$ plane. This is the flat sector $S b_{X X}$ collinear with the orange side:

In Figure 25, one shows how these 3 sectors make it possible to define an orthonormal basis of our sectoral space in which any sector can be decomposed in a unique way (with $\left.(x, y, z) \in \mathbb{R}^{3}\right)$. Each multiplicative coefficient $(x, y, z)$ Each multiplicative coefficient $(x, y, z)$ lengthens the base square sector into a rectangular sector:

$$
S 1=x S b_{X X}+y S b_{X Y}+z S b_{X Z}
$$

Note: In a $2 \mathrm{D}$ space, one retrieves the decomposition in real and imaginary pure part, $S 1=x S b_{X X}+y S b_{X Y}$ (with $(x, y) \in \mathbb{R}^{2}$ ) for which $S b_{X X}=1$ is the real unit vector (which becomes the flat sector in sectoral space) and $S b_{X Y}=i$ the pure imaginary unit vector (which becomes the square sector of $X Y$ plane).

Base according to the primality decomposition (multiplicative decomposition): 


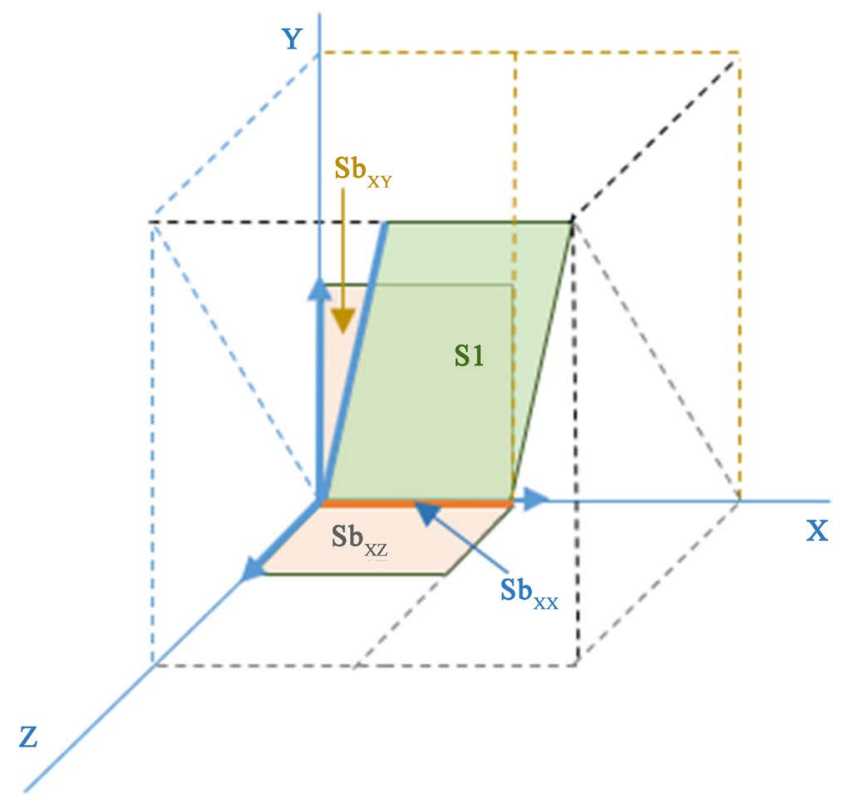

Figure 24. Two base sectors according to sectoral addition defining sectors with blue side in $Y Z$ plane.

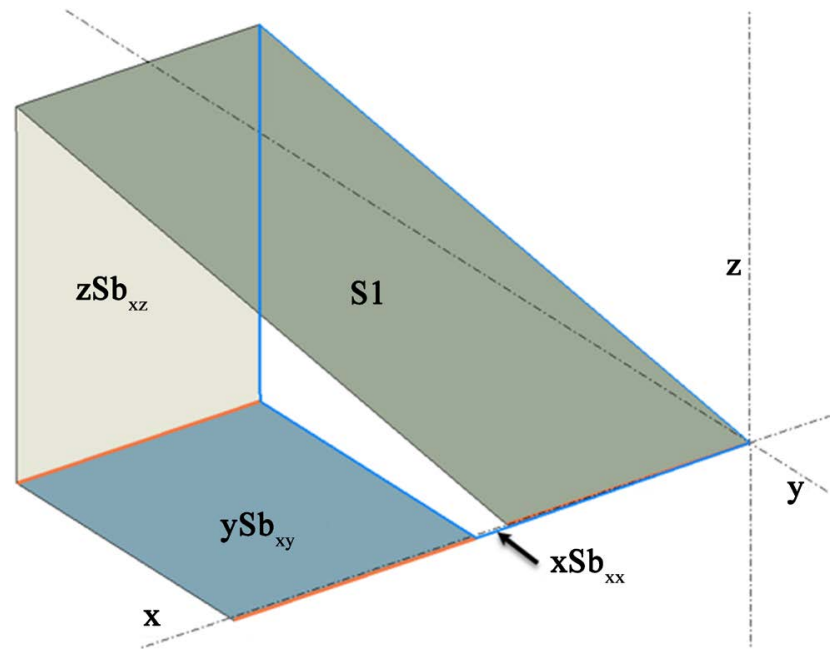

Figure 25. Base definition according to sectoral addition.

The preceding decomposition is based on the definition of sector addition, by which we make the orange sides coincide. But we can get a primality decomposition with the same basic sectors by following the definition of sector multiplication, by which we make the orange and blue sides coincide. In Figure 26, we have taken the same sector $S 1$ and the same basic square sectors. This time the sector is determined by exponents (plus a multiplicative coefficient associated with the flat unit sector). Each exponent "rotates" its base square sector, in its plane around the origin, transforming it into a rhombus. This has the consequence of positioning a blue "unit" side on the blue side of $S 1$. The multiplicative coefficient then makes it possible to give the size of the desired sector. Thus, $S b_{X Y}$ turns in its $X Y$ plane giving $S b_{X Y}^{t 1}$ and $S b_{X Z}$ turns in its $X Z$ plane giving $S b_{X Z}^{t 2}$ : 


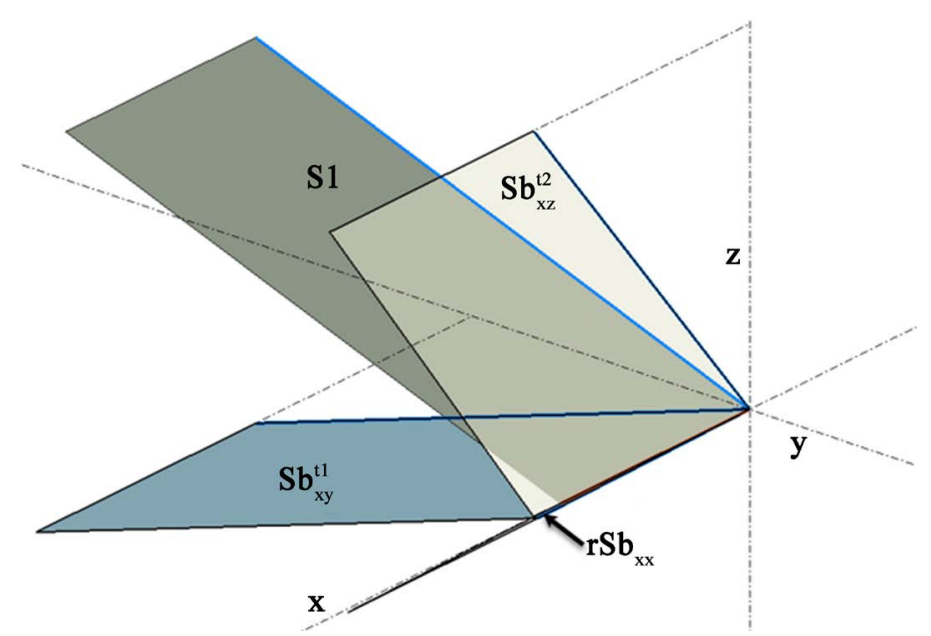

Figure 26. Base definition according to sectoral multiplication.

Note: In a 2D space, we retrieve the decomposition in its exponential form, $S 1=r S b_{X X} S b_{X Y}^{t 1} \quad$ (with $(r, t 1) \in \mathbb{R}^{2}$ and $t 1$ determined at a multiple close because related to the cyclicity of the rotation) for which $S b_{X X}=1$ is the real unit vector (which becomes the flat sector in the sector space) and $S b_{X Y}=i=\mathrm{e}^{\frac{i \pi}{2}}$ the pure imaginary unit vector (which becomes the square sector of the XY plane). In this writing, the angle of rotation of the blue side of the base sector $S b_{X Y}$ is $\frac{\pi}{2} t 1-\frac{\pi}{2}=\frac{\pi}{2}(t 1-1)$. We subtract $\frac{\pi}{2}$ from the angle because in the polar formulation of complex numbers, this angle is measured from the orange side.

\section{Dimension:}

For each new dimension, a new square sector must be added which defines the new plane, the new planar dimension (of which a vector is the orange side). We also note that any sectoral subspace consists at least of the flat sector $S b_{X X}$ and a square sector of another plane. In other words, any sectoral subspace always breaks down at least into a real part and a pure imaginary part. One thus finds in each of the subspaces an elongation (multiplicative coefficient) and one or more rotations (exponentiation coefficient), the latter being determined with a multiple close because related to the cyclicity of the rotations). We therefore find the existence several determinations (monodromy). We thus notice that these expressions are certainly systematically associated with functions close to the functions of circular type (trigonometric functions, elliptic functions ...).

Note: With this reflection on the concept of base, we note that to be more general the set of numbers associated with the sector space should be less the space of complexes than a space of number $T \times T=T^{2}$ which is a non-linear field. The components of the sectors then being numbers of $T$. In our article, we studied the case $T=\mathbb{R}$ whose $\mathbb{R}^{2}$ is related to complex numbers.

\subsubsection{Projective Space and Sectoral Space}

Our sectoral space is linked to projective spaces. Indeed, our sector can represent a projection between projective lines and conversely any projection between 
projective lines can be defined by a sector. A projection is defined by a projection center and 2 lines which sends each point from the start line to the finish line. It is enough to know the application on 3 points to define it [5]. Let's see concretely, how projection and sector are equivalent. In Figure 27, a projection has been shown from the red line $d 1$ to the blue line $d 2$ with its projection center "C". By taking the points of intersection $P 1$ and $P 2$ of the lines coming from the center of projection ("C") parallel to the two projective lines on which the projection is defined, we obtain 2 elements of definition for this projection which one will note" $f$ ":

$$
f\left(\infty_{d 1}\right)=P 2 \text { et } f(P 1)=\infty_{d 2}
$$

And finally, the point of intersection allows to define in a unique way the projection: $f(0)=0$.

Then, by the procedure already used to obtain the linked sectors, a translation which makes the point of intersection of the 2 projective lines coincide with the origin point of our sectoral space, then a zoom and a rotation to make both the projective start line coincide on the orange side ( $1^{\text {st }}$ vector of our base) and at the same time the point $P 1$ with the end of the orange unit vector, our projection thus defined represents a sector of our space. The elements which represent the sector thus take on a particular meaning: the orange side represents the projective line of departure, its end point $P 1$ such that $f(P 1)=\infty_{d 2}$, the blue side represents the projective line of arrival, its end point $P 2$ such that $f\left(\infty_{d 1}\right)=P 2$, the point of origin which makes this application a projection $f(0)=0$ and finally the last end of the sector which defines the centers of the projection. The special cases of flat sectors then correspond to the projections defined by two parallel lines $(d 1 / / d 2)$. The set of these particular cases in plane space is reduced by translation, rotation and zoom to the set of equivalence classes of blue vectors varying only on a line (in this case collinear with the orange vector). Thus, the projective space is to the sectoral space what the affine space is to the vector space. Indeed, an affine space can be seen as a vector space without a specific point of origin. Projective space in our case can be seen as a sectoral space with neither specific point of origin neither specific scale. The projective space is

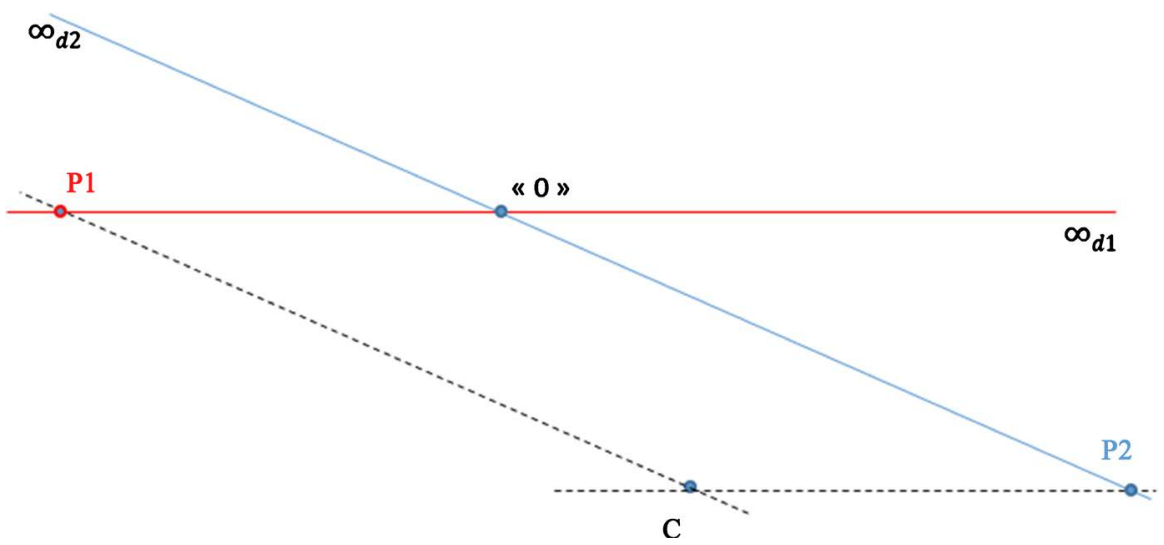

Figure 27. Sector and projection. 
the space of homographies and the sectoral space the space of projections, as the affine space is the space of affine applications and the vector space the space of linear applications.

Notes: The linear applications are the lines which turn around the origin, in our case the sectoral applications are the planes which turn around the "orange" side axis. We can also note that if the dimension of the affine space is $\mathrm{N}$ then the dimension of the associated vector space is $\mathrm{N}$ and the "intermediate" element (the vector) is of dimension 1 . Similarly in our case, if the dimension of the projective space is $\mathrm{N}$ then the dimension of the associated sectoral space is equivalent to the dimension of the associated vector space either $(\mathrm{N}+1)$ but the "intermediate" element (the sector) is of dimension 2. The increase of an order of the dimension of the sectoral space is compensated by a basic element of dimension also of a higher order.

\subsection{From a Binary Structure of Linear Group to a Ternary Structure of Non-Linear Group}

As we can expect from the definition of non-linear group, this implies no longer a binary distinction as in the linear group but a ternary distinction. The source of this ternary division appears in the notion of projection which, as we have just seen, is closely linked to our sectoral space. In Figure 28, a projection of center " $\mathrm{C}$ " is shown from the red line to the blue right. The green line is the line by which the point and its projection is equidistant from the point of intersection of the 2 lines (i.e. parallel to the external bisector of the 2 lines passing through "C"). On one side of the green line, the point P1 is more distant from " 0 " than $\mathrm{P} 0$, while on the other side P3 is closer than P2 to " 0 ". And similarly, on the other side of " 0 ", we still continue to have P5 closer to " 0 " than P4, but in the latter case there was an inversion of the distances at point " 0 ". Whereas in the linear approach (ring or field), the division of the multiplicative group of ] $0 ;+00$ [ around 1 is replicated symmetrically beyond 0 (by the additive group) forming a double binarity (“(+, +) |-1|(+,-) |0|(-, +) | $1 \mid(-,-)$ ”) in which these 4 domains are independent (without overlapping), here it is as if there always was

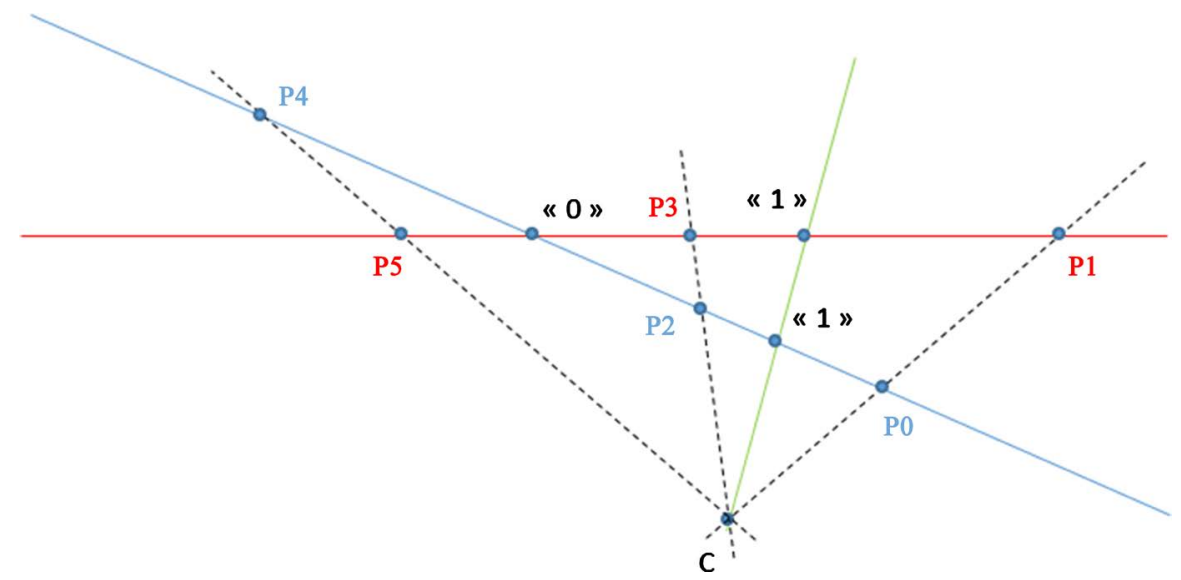

Figure 28. Ternary structure of non-linear group. 
these 2 divisions of the multiplicative group of ] $0 ;+00$ [ but this time the second division is symmetrized around " $1 / 2$ " (and not " 0 ") causing an overlap between " 0 " and " 1 " giving a triptych ( $\{+,+\}|0|\{+,-\}|1|\{-,-\}$ ”). This symmetrization around " $1 / 2$ " comes from the association of the 2 operations in writing $\forall z$ $\exists z_{1}$ telque $z=z_{1}\left(e-z_{1}\right)$.

But then, in these new structures, the objects become "thick", any element being defined by a combination of the 2 operations ( $\forall z, \exists z_{1} z=z_{1}\left(e-z_{1}\right)$ ), they mix. And mixture cannot appear neither in a punctual representation, nor in the relation between two punctual representations idealized by a vector (only a surface element, "thick" object, can idealize a mixture). One could criticize that this ternary division is nevertheless less fine than that doubly binary of the field or ring. But the advent of this thickening and this mixing takes place in our structures at the more fundamental level of the group and generates the possibility of self-similarity, replication at different scales of this ternary diagram (as the logistic sequence $f\left(x_{n+1}\right)=x_{n}\left(1-x_{n}\right)$ ), like the baker's transformation (lengthening and folding) involved in the study of dynamic systems [6]. This harmonious tangle is ensured by the rules of distributivities of the nonlinear field. The nonlinear group and its ternary division thus generate an infinitely finer modeling, more nuanced than the binary domain.

\section{Conclusions}

In this study, we have proposed 3 new nonlinear algebraic structures which extend the linear notions of group, field and vector space. Geometrically, sectorial space, analytically, the set of complex numbers and algebraically, the pairs of complex vectors are 3 sets studied in this article which verify these 3 structures of nonlinear space (sectorial space), of nonlinear field and of nonlinear group.

The first fundamental characteristics which emerge from these structures are the notions of extended object and ternary structure (“ $\{+,+\}|0|\{+,-\}|1|\{-$, $-\}$ ") through the complementary association which appears in the definition of the nonlinear group, $\forall z \quad \exists z_{1}$ such that $z=z_{1}\left(1-z_{1}\right)$. These notions extend and "qualify" what the linear group depicts with its punctual objects and its binary structure (“+ $|0|-$ "). The segment/vector becomes parallelogram/sector, the minimum dimension of space " $1 \mathrm{D}$ " becomes " $2 \mathrm{D}$ ", the opposites/inverses become association/mixture of complementarities, the single center is deployed in 2 focuses, circle and line of the vector space are transformed into the 3 conics, from linear equations we go to $2^{\text {nd }}$ degree equations, from translations we go to projections, at invariance by translation is added invariance by zoom and rotation ...

This complementary association " $z_{1}\left(1-z_{1}\right)$ " allows to glimpse its full potential in the light of the dynamic systems of which this form is the echo of the logistic sequence (mixing, transformation of the baker, iteration, pattern of period doubling, fractal, chaos, ...). And this ability to process mixtures leads us to 
phase transitions, renormalization groups, etc. Because these structures are in relation to the projective spaces, they could appear at the center of a very large set of domains.

One of the foundations of quantum mechanics is the existence of conjugate parameters that do not commute. A parameter cannot be known as precisely as one wants without losing knowledge about the conjugate parameter. However, a sector characterizes an extended object (irreducible to a point) and this irreducibility is conceptually close to the behavior of these conjugated parameters. It correlates two degrees of freedom which cannot be defined independently.

Current theoretical physics is based on very general principles of invariance. If we perform an experiment, we must be able to describe it in the same way regardless of the location and orientation in the laboratory (translational and rotational invariance). However, it would be quite relevant to be able to describe this same experience whether you are giant or tiny. In other words, the laws of physics should also be zoom invariant. Note that at present, general relativity schematically models large scales and quantum mechanics models small scales and that these two theories are irreconcilable. One of the major themes of current theoretical physics is to try to reconcile these two models. To this goal, the sectoral space could be the appropriate mathematical tool.

To end with a speculative but also very exciting perspective, one can notice that this work is, from a certain point of view, in the continuity of the evolution of mathematics. Very schematically, we could say that at order 0 , mathematical theories are based on the strict equality of entities: " $a=b$ ". At order 1, more general mathematical theories are based on the equality of the ratio of entities: " $a / b=c / d$ ". Our theory would then define a theory of order 2 based on the double-ratio equality of entities: " $(a / b) /(c / d)=(e / f) /(g / h)$ ". Note also that, once again very schematically, these equalities (through the intermediary of the equivalence classes) are also associated with sets of numbers, the natural numbers for the $1^{\text {st }}$ equality, the rational for the second. We can then wonder if equivalence classes based on the double-ratio would not give rise to a new set of "projective" numbers. One could then also wonder if this set would be equivalent to algebraic or transcendent numbers. Unless in the same way as between natural numbers and rational numbers, an intermediate set (associated with the notion of group) appears (integers), an intermediate set between rational and projective numbers (associated with the notion of nonlinear group) would appear and which would perhaps be algebraic or other numbers ...

\section{Conflicts of Interest}

The author declares no conflicts of interest regarding the publication of this paper.

\section{References}

[1] Dixmier, J. (1993) Cours de mathématiques du premier cycle. Bordas Editions. 
[2] Flament, D. (2003) Histoire des nombres complexes. CNRS Editions.

[3] Hruza, J. and Trachsler, M. (2019) The Modular Group and the Fundamental Domain. Seminar on Modular Forms Spring 2019, Zürich, 13th March 2019, 1-10.

[4] Duverney, D. (1998) Théorie des nombres. Dunod.

[5] Sidler, J.C. (2000) Géométrie projective. Dunod.

[6] Bergé, P., Pomeau, Y. and Vidal, C. (1988) L'ordre dans le chaos. Hermann. 